\title{
Menin inhibitor MI-3454 induces remission in MLL1-rearranged and NPM1-mutated models of leukemia
}

\author{
Szymon Klossowski, ${ }^{1}$ Hongzhi Miao, ${ }^{1}$ Katarzyna Kempinska, ${ }^{1}$ Tao Wu, ${ }^{2}$ Trupta Purohit,, EunGi Kim, ${ }^{1}$ Brian M. Linhares, \\ Dong Chen, ${ }^{1}$ Cloria Jih, ${ }^{3}$ Eric Perkey, ${ }^{3}$ Huang Huang, ${ }^{1}$ Miao He, ${ }^{4}$ Bo Wen, ${ }^{4}$ Yi Wang, ${ }^{2}$ Ke Yu, ${ }^{2}$ Stanley Chun-Wei Lee, ${ }^{5}$ \\ Gwenn Danet-Desnoyers, ${ }^{6}$ Winifred Trotman, ${ }^{6}$ Malathi Kandarpa, ${ }^{7}$ Anitria Cotton, ${ }^{8}$ Omar Abdel-Wahab, ${ }^{5}$ Hongwei Lei, ${ }^{1}$ \\ Yali Dou, ${ }^{1}$ Monica Guzman, ${ }^{9}$ Luke Peterson, ${ }^{7}$ Tanja Gruber, ${ }^{8}$ Sarah Choi, ${ }^{1}$ Duxin Sun, ${ }^{4}$ Pingda Ren, ${ }^{2,10}$ Lian-Sheng Li, ${ }^{2}$ \\ Yi Liu, ${ }^{2}$ Francis Burrows, ${ }^{10}$ Ivan Maillard, ${ }^{3,6}$ Tomasz Cierpicki, ${ }^{1}$ and Jolanta Grembecka'
}

'Department of Pathology, University of Michigan, Ann Arbor, Michigan, USA. ${ }^{2}$ Wellspring Biosciences, Inc., San Diego, California, USA. ${ }^{3}$ Life Sciences Institute and ${ }^{4}$ College of Pharmacy, University of Michigan, Ann Arbor, Michigan, USA. ${ }^{5}$ Memorial Sloan Kettering Cancer Center, New York, New York, USA. ${ }^{6}$ Division of Hematology-Oncology, Department of Medicine, Perelman School of Medicine, University of Pennsylvania, Philadelphia, Pennsylvania, USA. 'Division of Hematology/Oncology, Department of Internal Medicine, University of Michigan, Ann Arbor, Michigan, USA. ${ }^{8}$ Saint Jude Children's Hospital, Memphis, Tennessee, USA. ${ }^{9}$ Weill Cornell Medicine, New York, New York, USA. ${ }^{10}$ Kura Oncology, Inc., San Diego, California, USA.

\begin{abstract}
The protein-protein interaction between menin and mixed lineage leukemia 1 (MLL1) plays a critical role in acute leukemias with translocations of the MLL1 gene or with mutations in the nucleophosmin 1 (NPM1) gene. As a step toward clinical translation of menin-MLL1 inhibitors, we report development of MI-3454, a highly potent and orally bioavailable inhibitor of the menin-MLL1 interaction. MI-3454 profoundly inhibited proliferation and induced differentiation in acute leukemia cells and primary patient samples with MLL1 translocations or NPM1 mutations. When applied as a single agent, MI-3454 induced complete remission or regression of leukemia in mouse models of MLL1-rearranged or NPM1-mutated leukemia, including patient-derived xenograft models, through downregulation of key genes involved in leukemogenesis. We also identified MEIS1 as a potential pharmacodynamic biomarker of treatment response with MI-3454 in leukemia, and demonstrated that this compound is well tolerated and did not impair normal hematopoiesis in mice. Overall, this study demonstrates, for the first time to our knowledge, profound activity of the menin-MLL1 inhibitor as a single agent in clinically relevant PDX models of leukemia. These data provide a strong rationale for clinical translation of MI-3454 or its analogs for leukemia patients with MLL1 rearrangements or NPM1 mutations.
\end{abstract}

\section{Introduction}

The development of acute leukemia is associated with heterogeneous genetic and epigenetic alterations $(1,2)$, making it difficult to identify new effective therapies for leukemia patients. Despite these challenges, numerous efforts have attempted to develop new

Authorship note: SK, HM, and KK contributed equally to this work. Conflict of interest: JC and TC received research support from Kura Oncology, Inc. They have also served as consultants for Kura Oncology, have equity ownership in the company, and are coinventors (along with SK, TW, LS, and PR) on patent applications covering MI-3454 (PCT/US2017/022535). PR is an employee of Kura Oncology, Inc. and has a significant ownership interest in the parent of Wellspring Biosciences, Inc. FB is an employee of Kura Oncology, Inc. Kura Oncology, Inc. and the University of Michigan have filed patent applications covering MI-3454 and they hold intellectual property rights on this compound. OAW has served as a consultant for H3B Biomedicine, Foun dation Medicine Inc, Merck, and Janssen, and has received prior research funding from $\mathrm{H} 3 \mathrm{~B}$ Biomedicine unrelated to the current manuscript. MC receives research support from Cellectis and serves as a consultant in SeqRx.

Copyright: () 2020, American Society for Clinical Investigation.

Submitted: March 26, 2019; Accepted: November 6, 2019; Published: December 19, 2019 Reference information: / Clin Invest. 2020;130(2):981-997.

https://doi.org/10.1172/JCl129126. therapeutic agents for acute leukemias, some of which are currently in clinical development (3-6). Translocations of the mixed lineage leukemia 1 ( $M L L 1$, also known as $M L L$ or KMT2A) gene, which are found in approximately $5 \%-10 \%$ of acute leukemia patients, both children and adults, result in an aggressive subtype of leukemia, with a 5-year survival rate of only approximately $35 \%$ $(7,8)$. MLL1 translocations are particularly prevalent in infant leukemias, constituting up to $80 \%$ of infant acute lymphoblastic leukemia (ALL) cases, which demonstrate a high level of resistance to chemotherapy $(9,10)$. MLL1 translocations lead to fusion of the $\mathrm{N}$-terminal fragment of $M L L 1$ with 1 of over 60 protein partners (11-13), resulting in the expression of MLL fusion proteins that enhance proliferation and block hematopoietic differentiation, ultimately driving the development of leukemia (14). Because of poor patient outcomes, MLL leukemia represents an unmet medical need, necessitating the development of new therapies.

The protein-protein interaction between menin and MLL fusion proteins plays a critical role in the pathogenesis of MLL leukemia, and the genetic disruption of this interaction abrogates the development of acute leukemia in vivo (15-17). Our 
A<smiles>CNc1nc(NC2CCN(Cc3ccc4c(c3)nc(NC)n4CC(C)(C)C)CC2)c(C#N)cc1CC(F)(F)F</smiles>

MI-3454
B

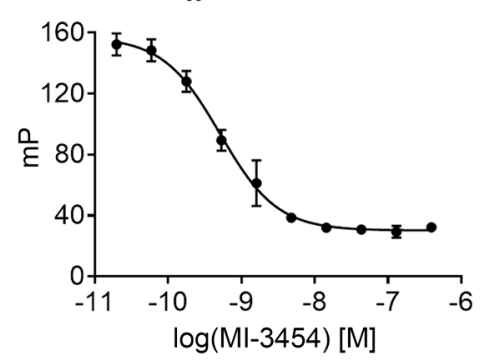

C

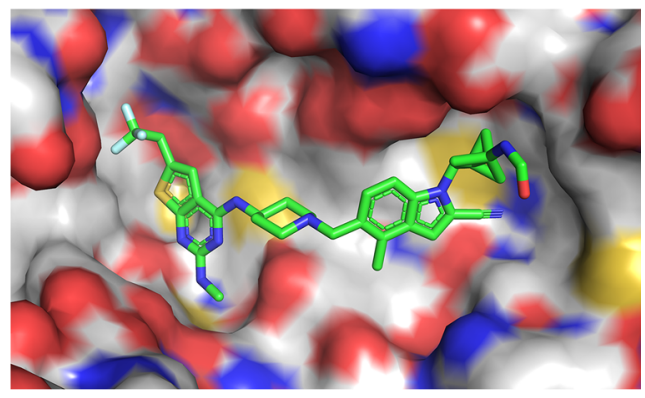

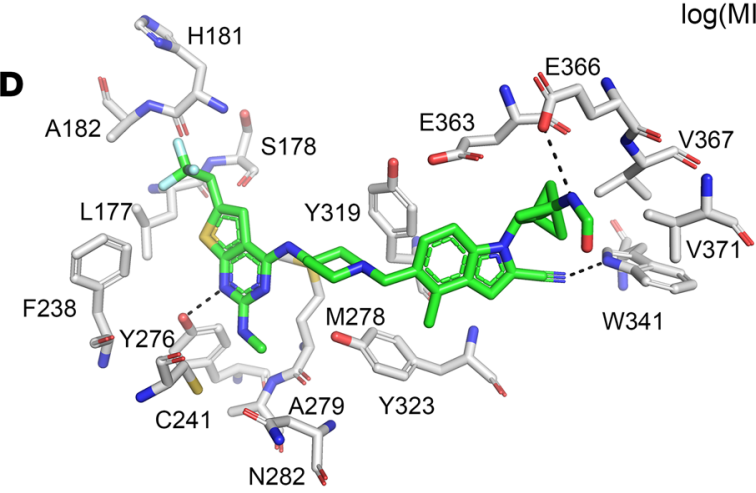

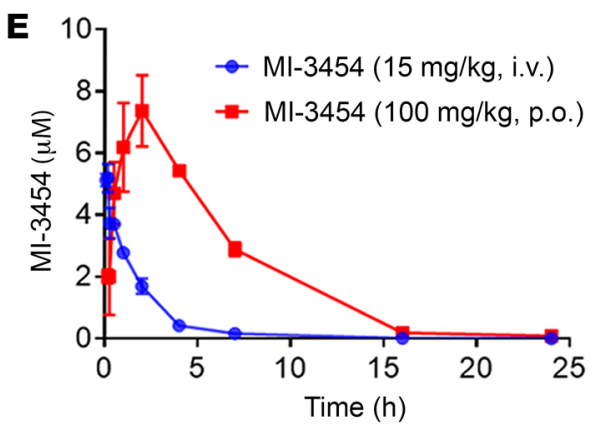

$\mathbf{F}$

\begin{tabular}{|l|r||l|l|}
\hline MLL-tr & $\mathrm{GI}_{50}(\mathrm{nM})$ & Non-MLL-tr & $\mathrm{GI}_{50}(\mathrm{nM})$ \\
\hline$\rightarrow$ MV-4-11 & $7.6 \pm 0.8$ & $\rightarrow$ REH & $2700 \pm 400$ \\
$\rightarrow$ MOLM13 & $12.5 \pm 0.9$ & $\rightarrow$ U937 & $>6000$ \\
$\rightarrow$ RS4-11 & $26.0 \pm 3.0$ & $\rightarrow$ SET2 & $>6000$ \\
$\rightarrow$ SEM & $24.9 \pm 2.6$ & $\rightarrow$ K562 & $>6000$ \\
$\rightarrow$ KOPN8 & $27.1 \pm 2.0$ & & \\
\hline
\end{tabular}
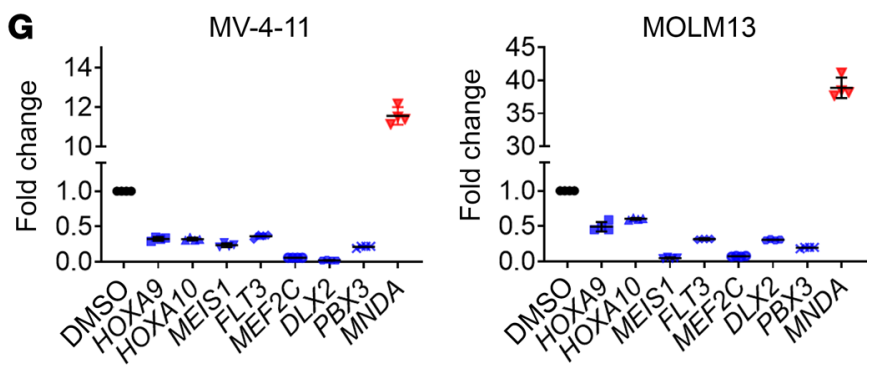

H

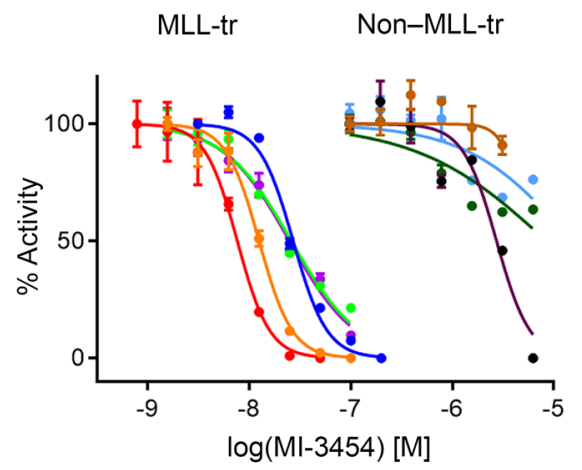

I

DMSO
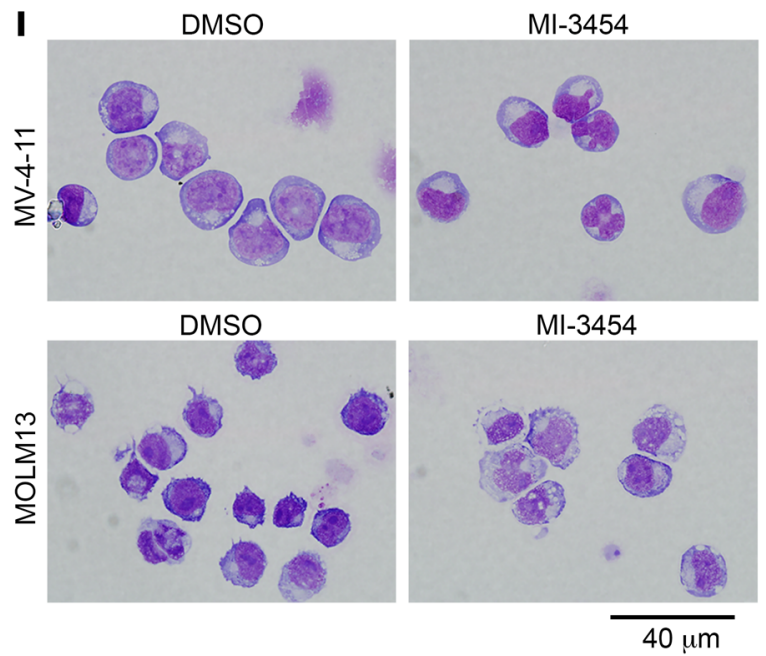

Figure 1. Structure and in vitro activity of menin-MLL1 inhibitor MI-3454. (A) Chemical structure of MI-3454. (B) Titration curve from fluorescence polarization competition assay for inhibition of the menin-MLL1 ${ }_{4-43}$ interaction by MI-3454. Mean $\pm \mathrm{SD}, n=3$. $\mathrm{mP}$, millipolarization. (C) Crystal structure of the menin-MI-3454 complex (1.24 Å). Menin is shown in surface representation (carbon atoms in gray, oxygen in red, nitrogen in dark blue, sulfur in yellow) and MI-3454 is shown in stick representation (carbon atoms in green; color coding for oxygen, nitrogen, and sulfur atoms is the same as for menin residues; fluorine atoms are in light blue). (D) Details of interactions of MI-3454 with menin. Color coding as in C. (E) Pharmacokinetic studies in mice performed for MI-3454 (mean \pm SD, $n=3$ ) demonstrating blood concentration of Ml-3454 after oral (p.o.) dose of $100 \mathrm{mg} / \mathrm{kg}$ and intravenous (i.v.) administration at $15 \mathrm{mg} /$ $\mathrm{kg}$. (F) Titration curves from MTT cell viability assay performed after 7 days of treatment of human MLL leukemic cell lines (MLL-tr) with MI-3454: MV-4-11 (MLL-AF4), MOLM-13 (MLL-AF9), KOPN-8 (MLL-ENL), SEM (MLL-AF4), RS4-11 (MLL-AF4), and control leukemic cell lines (non-MLL-tr): K562, SET2, REH, and U937; mean $\pm S D, n=4$. Two to 3 independent MTT experiments were performed for each cell line. Representative graphs are shown. $\mathrm{CI}_{50}$ values correspond to MI-3454 concentrations needed for 50\% inhibition of cell proliferation. (C) Quantitative RT-PCR performed in MV-4-11 cells (left) or MOLM13 cells (right)

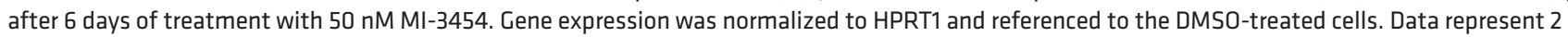
independent experiments, each performed in duplicate (mean $\pm \mathrm{SD}, n=4$ ). $P<0.0001$ for all genes tested calculated using Student's 2 -tailed $t$ test. (H) Wright-Giemsa-stained cytospins for MV-4-11 and MOLM13 after 6 days of treatment with 50 nM MI-3454. 
previous work has resulted in the development of first-in-class small-molecule inhibitors of the menin-MLL1 interaction, which directly bind to the MLL1 binding site on menin and block this protein-protein interaction (17-23). Because the menin binding motif is retained in all MLL fusion proteins, these compounds also block the interaction of menin with MLL fusions (22). The menin-MLL1 inhibitors that we developed previously, including MI-503 (17) and MI-1481 (21), demonstrate relatively robust activity and an on-target mechanism of action in the MLL-rearranged leukemic cells and delay leukemia progression in mouse models of MLL leukemia. However, the existing small-molecule inhibitors of the menin-MLL1 interaction require further optimization of their potency, selectivity, pharmacokinetic (PK) profile, and other drug-like properties before they can be translated to clinical studies in leukemia patients.

Recent studies have also revealed the importance of the menin-MLL1 wild-type interaction in acute myeloid leukemia (AML) with mutations in the nucleophosmin 1 (NPM1) gene (24). NPM1 mutations are found in over $30 \%$ of AML patients $(25,26)$ and are associated with the upregulated expression of $H O X A$, $H O X B$, and MEIS1 genes $(24,27,28)$. Mutations in NPM1 co-occur with mutations in other AML driver oncogenes, such as FLT3 (in $\sim 60 \%$ of cases, including FLT3-internal tandem duplication [FLT3ITD]) and/or DNMT3A (in $50 \%$ of cases) (29-31). Coexistence of the FLT3-ITD and NPM1 mutations defines the most unfavorable category of NPM1-mutated AMLs (30), with an overall survival rate below $50 \%(31,32)$, emphasizing the need for new therapies. Previous studies have demonstrated growth arrest and differentiation of AML cells with NPM1 mutations upon treatment with the previous generation of menin-MLL1 inhibitors (MI-503 and MI-2-2) (24). However, high concentrations of these compounds $(>2 \mu \mathrm{M})$ were required to affect gene expression in the NPM1-mutated AML cells, and the in vivo effect was relatively modest (24). These results further support the need for the development of more potent meninMLL1 inhibitors with optimized drug-like properties.

The development of small-molecule inhibitors targeting the menin-MLL1 interaction has proven challenging because of the high potency and the bivalent nature of this interaction, which involves 2 MLL1 fragments ( MLL1 $_{4-15}$ and MLL1 $\left.1_{23-40}\right)$ (33). Thus, a menin-MLL1 inhibitor needs to occupy a relatively large area of the binding site for effective inhibition of this protein-protein interaction (21), resulting in an increase in the molecular weight of such compounds that leads to challenges in optimizing their drug-like properties. Here, we report the development of a highly potent (subnanomolar), very selective, and orally bioavailable inhibitor of the menin-MLL1 interaction, MI-3454, with a profound activity in leukemic cells and primary patient samples with $M L L 1$ rearrangements or NPM1 mutations. We demonstrate that MI-3454 as a single agent induces complete remission or regression in the MLL1-rearranged and NPM1-mutated leukemia models, including patient-derived xenograft (PDX) models. We also provide insights into the mechanistic aspects of pharmacological inhibition of the menin-MLL1 interaction in the NPM1-mutated AMLs and identify MEIS1 as a pharmacodynamic biomarker of treatment response to the menin-MLL1 inhibitor in leukemia. Overall, this work provides a strong rationale for clinical translation of the menin-MLL1 inhibitor to acute leukemia patients with $M L L 1$ translocations or NPM1 mutations.

\section{Results}

Development of a highly potent menin-MLL1 inhibitor with subnanomolar inhibitory activity. We have previously provided proof of concept that inhibition of the menin-MLL1 interaction by the small-molecule inhibitor MI-503 (Supplemental Figure 1; supplemental material available online with this article; https://doi.org/10.1172/ JCI129126DS1) delays the progression of leukemia in vivo (17). Here, we used the crystal structure of menin in complex with MI-503 (4X5Y in PDB) to design new analogs with improved inhibitory activity and optimized drug-like properties. These efforts resulted in the development of MI-3454 (Figure 1A), which was obtained by substituting the thienopyrimidine ring with the $\mathrm{N}$-methyl amino group and by introducing at the indole nitrogen the methyl-bicyclo[1.1.1]pentane group that harbors a terminal amide. Remarkably, MI-3454 demonstrates subnanomolar inhibitory activity $\left(\mathrm{IC}_{50}=0.51 \mathrm{nM}\right)$ in blocking the interaction of menin with an $\mathrm{MLL1}_{4-43}$ fragment encompassing the entire menin binding motif, representing a nearly 60-fold improvement over MI-503 (Figure 1B and Supplemental Figure 1).

To reveal the molecular basis of the inhibition caused by MI-3454, we solved the crystal structure of menin in complex with MI-3454 (Figure 1, C and D, and Supplemental Table 1). The structure demonstrates that MI-3454, which binds to the MLL1 binding site on menin, achieves a high level of shape complementarity with the protein (Figure 1C), which likely contributes to the superior inhibitory activity of this compound. The MI-3454 compound is involved in 3 hydrogen bonds with Try276, Trp341, and Glu366 on menin as well as in hydrophobic interactions (Figure 1D). The $\mathrm{N}$-methyl-amino group in MI-3454, which is absent in MI-503, fits very well into the small cavity formed by the side chains of Met278, Cys241, Tyr276, and Ala279 (Figure 1D). Furthermore, the methyl-bicyclo[1.1.1]pentane group introduced at the indole nitrogen of MI-3454 is engaged in hydrophobic interactions with menin, including Val367, Val371, and Glu366, while the terminal amide is involved in a hydrogen bond with Glu366 (Figure 1D).

To assess the utility of MI-3454 as a candidate for in vivo studies and potential clinical translation, we assessed the druglike properties of this compound. First, we found that MI-3454 exhibits favorable stability in murine and human liver microsomes $\left(t_{1 / 2}=20.4\right.$ minutes and 37.1 minutes, respectively) (Supplemental Figure 2A). Furthermore, MI-3454 did not potently inhibit cytochromes $\mathrm{P} 450(<50 \%$ inhibition at $10 \mu \mathrm{M}$ MI-3454 for most of them) (Supplemental Figure 2B). In addition, the aqueous solubility of MI-3454 is acceptable and exceeds $15 \mu \mathrm{M}$. Subsequent PK studies of MI-3454 in mice revealed a high exposure in plasma (AUC $=32,631 \mathrm{~h} \cdot \mathrm{ng} / \mathrm{mL}$ ) upon oral administration, suitable halflife (3.2 hours), and high oral bioavailability ( 77\%) (Figure 1E and Supplemental Figure 2C). MI-3454 demonstrates, however, lower levels in brain and cerebrospinal fluid, suggesting limited ability to cross the blood-brain barrier (Supplemental Figure 2D). Overall, MI-3454 is a very potent menin-MLL1 inhibitor with favorable drug-like properties that outperforms previously reported inhibitors of this protein-protein interaction, representing a valuable candidate for evaluation in advanced models of leukemia.

MI-3454 demonstrates pronounced activity and on-target mechanism of action in MLL leukemic cells. We then tested the activity of MI-3454 in a panel of leukemic cell lines with and without MLL1 translocations. We found that MI-3454 markedly reduced the 
A

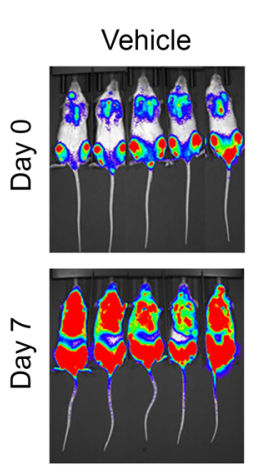

C Peripheral blood

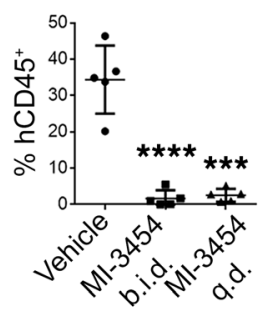

$\mathrm{MI}-3454$

$120 \mathrm{mg} / \mathrm{kg}$, q.d. $120 \mathrm{mg} / \mathrm{kg}$, b.i.d.
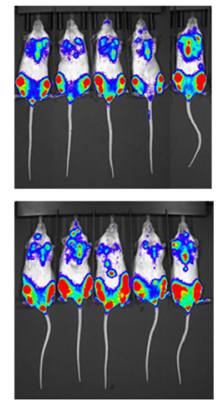

Bone marrow

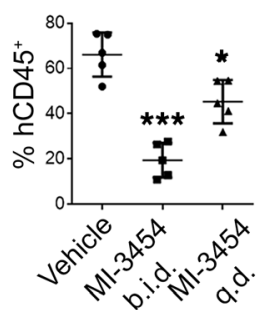

$\mathrm{MI}-3454$

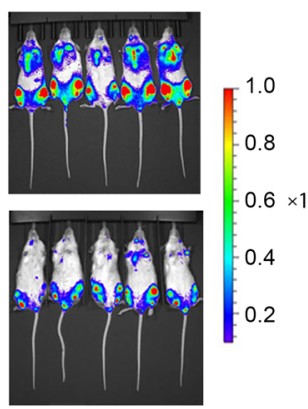

Spleen

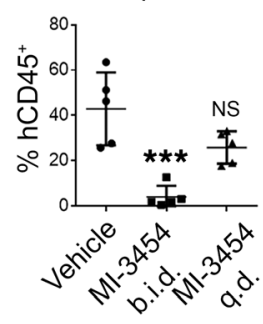

E
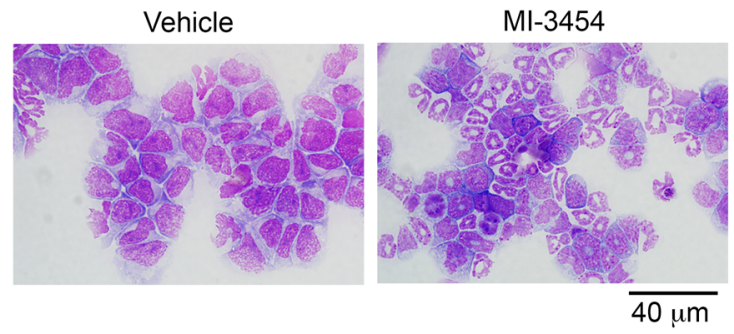

B

- Vehicle

- MI-3454 (120 mg/kg, b.i.d.)

- MI-3454 (120 mg/kg, q.d.)

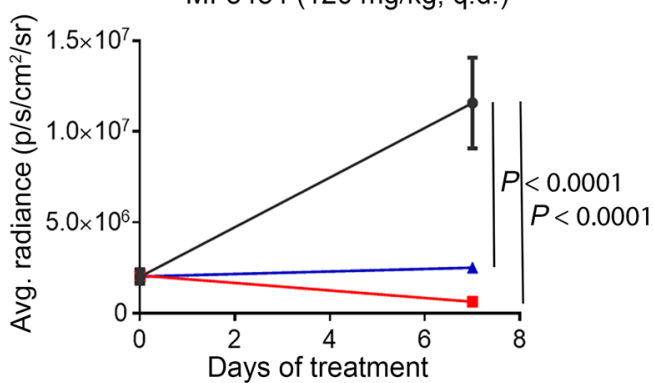

D

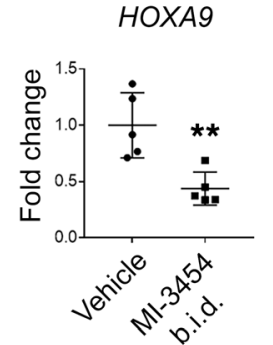

MEIS1
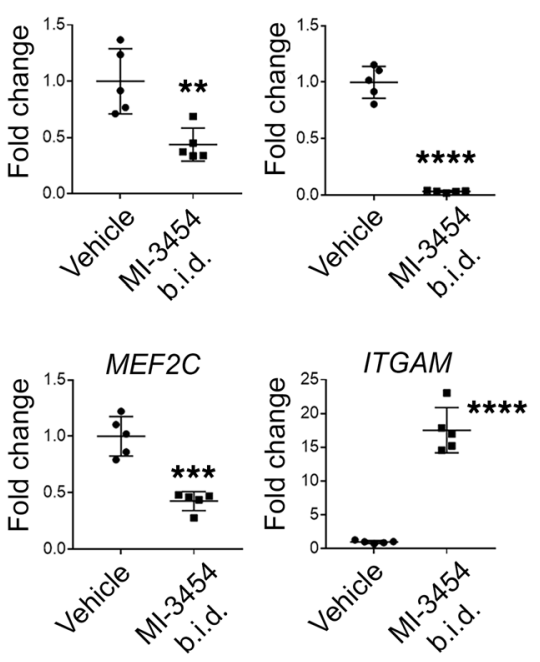
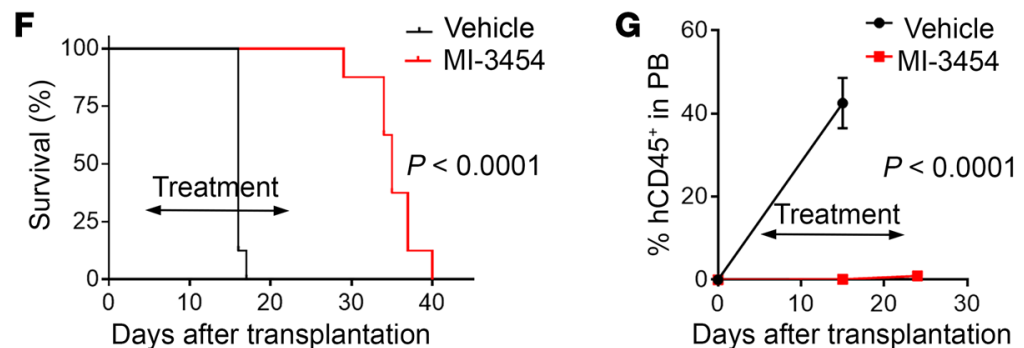

Figure 2. In vivo activity of MI-3454 in xenograft models of MLL leukemia. (A) Bioluminescent imaging of NSC mice transplanted with MV-4-11 human MLL leukemic cells expressing luciferase performed on the indicated days after initiation of treatment with MI-3454 or vehicle, $n=5$. (B) Quantification of bioluminescence level in MV-4-11 mice on the indicated days after initiation of treatment with MI-3454 or vehicle (mean \pm SEM, $n=5$ ). (C) Flow cytometric quantification of human $\mathrm{CD} 45^{+}$cells in peripheral blood, spleen, and bone marrow samples harvested from MV-4-11-transplanted mice after 7 days of treatment with MI-3454 (120 mg/kg, b.i.d., p.o. or $120 \mathrm{mg} / \mathrm{kg}$, q.d., p.o.) or vehicle; mean \pm SD, $n=5$. (D) Gene expression measured by qRT-PCR of RNA extracted from bone marrow samples harvested from MV-4-11 mice after 7 days of treatment with MI-3454 (120 mg/kg, b.i.d., p.o.) or vehicle. Transcript levels are normalized to HPRT1 and referenced to the mean transcript level in the vehicle-treated group, which is set as $1(n=5)$. In $\mathbf{C}$ and $\mathbf{D},{ }^{*} P<0.05$; ${ }^{* *} P$ $<0.01$; ${ }^{* *} P<0.001$; ${ }^{* * *} P<0.0001$; NS, not significant; calculated using 2-way ANOVA with Tukey's post hoc test (C) or Student's 2-tailed $t$ test (D). (E) Wright-Giemsa-stained cytospins for bone marrow samples isolated from MV-4-11 mice after 7 days of treatment with MI-3454 (120 mg/kg, b.i.d., p.o.) or vehicle. (F) Kaplan-Meier survival curves of vehicle- and MI-3454-treated (100 mg/kg, b.i.d., p.o.) NSG mice transplanted with MOLM13 cells ( $n=8$ ). $P$ value was calculated using log-rank (Mantel-Cox) test. (C) Flow cytometric quantification of human CD45+ cells in peripheral blood (PB) of MOLM13 mice during treatment with MI-3454 or vehicle. Mean \pm SEM, $n=8$. $P$ values in B and $\mathbf{G}$ were calculated using 2-way ANOVA with Sidak's multiple-comparisons test.

viability of leukemic cells harboring various MLL fusion proteins (MLL-AF9, MLL-AF4, MLL-ENL), with $\mathrm{GI}_{50}$ values ranging from 7 to $27 \mathrm{nM}$ (Figure $1 \mathrm{~F})$. This reveals the superior activity of MI-3454 in MLL leukemic cells over all menin-MLL1 inhibitors reported to date (17-21, 34). In contrast, MI-3454 did not significantly affect the growth of leukemic cells without MLL1 translocations, demonstrating over 100-fold selectivity toward MLL leukemic cells (Figure 1F).

We then assessed the effect of MI-3454 on the expression level of the MLL fusion target genes in 2 MLL leukemic cell lines, MV-4-11 and MOLM13. We found that nanomolar concentrations 
of this compound (50 nM or lower) led to downregulated expression of HOXA9 and MEIS1, 2 genes that are critical for MLL fusion proteins to mediate leukemogenesis (Figure $1 \mathrm{G}$ and Supplemental Figure 3, A and B). Furthermore, the expression level of other MLL fusion target genes, including MEF2C, DLX2, HOXA1O, $P B X 3$, and FLT3 was also substantially reduced upon treatment with MI-3454 (Figure 1G and Supplemental Figure 3, A and B). Downregulated expression of the MLL fusion target genes was accompanied by differentiation in both MLL leukemic cell lines upon treatment with MI-3454, as shown by a substantial increase in the expression level of the MNDA differentiation marker and by morphological change in these cells (Figure 1, G and $\mathrm{H}$, and Supplemental Figure 3, A and B). Importantly, exogenous overexpression of HOXA9 in the MOLM13 MLL leukemic cell line (HOXA9-MOLM13) led to limited cell growth inhibition and had no effect on HOXA9 expression upon treatment with MI-3454 (Supplemental Figure 4, A-C; see complete unedited blots in the supplemental material). This indicates that overexpression of HOXA9 reverses the effects of MI-3454, validating the on-target mechanism of action of this compound in MLL leukemic cells. Furthermore, MI-3454 demonstrated strong reduction of cell proliferation and downregulation of HOXA9 and MEIS1 expression in mouse bone marrow cells transformed with the MLL-AF9 oncogene but not in bone marrow cells transformed with HOXA9 and MEIS1 oncogenes (Supplemental Figure 4, D-F). These data further support the idea that overexpression of HOXA9 and MEIS1 through a mechanism irrespective of MLL fusions abolished the effect of MI-3454 in leukemic cells. Taken together, these results show that MI-3454 demonstrates very pronounced activity and an on-target mechanism of action in MLL leukemic cells, supporting its utility for in vivo studies.

$M I-3454$ induces leukemia regression in a xenograft model of $M L L$ leukemia through on-target activity. To assess the in vivo efficacy and on-target activity of MI-3454, we utilized the MV-4-11 xenotransplantation model of MLL leukemia. MV-4-11 cells (harboring MLL-AF4) expressing luciferase were transplanted into NSG mice to induce systemic leukemia, and disease progression was monitored by measuring the bioluminescence signal in live animals. To test the effect of MI-3454 in the context of advanced leukemia, treatment with MI-3454 or vehicle [20\% (2-hydroxypropyl)- $\beta$-cyclodextrin, 5\% Cremophor in water] was initiated 19 days after the transplantation of the MV-4-11 cells into the mice, when the level of leukemic blasts in the bone marrow, peripheral blood, and spleen had reached approximately $30 \%, 7 \%$, and 6\%, respectively (Supplemental Figure 5A). MI-3454 was administered orally at $120 \mathrm{mg} / \mathrm{kg}$ once or twice daily for 7 consecutive days, and it did not show any negative impact on mouse body weight (Supplemental Figure 5B). As evidenced by the bioluminescence levels, a once-daily treatment with MI-3454 was sufficient to block leukemia progression when compared with the vehicle-treated mice (Figure 2, A and B). Remarkably, we observed marked regression of leukemia after twice-daily administration of MI-3454, as indicated by a substantially reduced bioluminescence signal in MV-411 mice treated with MI-3454 as compared with the pretreatment status or vehicle-treated mice (Figure 2, A and B). Furthermore, an analysis of bone marrow, blood, and spleen samples isolated from MV-4-11 mice demonstrated a markedly reduced level of leukemic blasts (hCD45+ cells) in the MI-3454-treated mice as compared with the vehicle control (Figure $2 \mathrm{C}$ and Supplemental Figure 5C). Importantly, the twice-daily treatment of mice with MI-3454 resulted in a lower level of leukemic blasts as compared with the pretreatment status, providing further evidence of leukemia regression (Figure $2 \mathrm{C}$ and Supplemental Figure $5 \mathrm{~A}$ ) and in agreement with bioimaging data (Figure 2, A and B).

To validate the on-target in vivo activity of MI-3454, we performed gene expression studies in bone marrow samples isolated from mice treated with MI-3454 or the vehicle. A marked reduction (>30-fold) in the expression level of MEIS1, the MLL fusion target gene, was observed upon treatment of mice with MI-3454 versus vehicle-treated mice (Figure 2D). The expression level of other MLL fusion target genes, HOXA9 and MEF2C, was also substantially reduced in the MI-3454-treated mice (Figure 2D). Furthermore, a pronounced ( $>5$-fold) increase in expression of the differentiation marker ITGAM was observed following the treatment of mice with MI-3454, accompanied by a differentiating phenotype of bone marrow cells (Figure 2, D and E, and Supplemental Figure 5D). Altogether, MI-3454 demonstrates very pronounced in vivo activity, manifested by leukemia regression in the MLL1-rearranged leukemia xenograft model of advanced disease. This correlates with a pronounced downregulation of MEIS1 expression, which might represent a valid biomarker for assessing the effectiveness of menin-MLL1 inhibitors in future clinical studies.

MI-3454 markedly prolongs survival of MLL leukemia mice. Next, we sought to assess the effect of MI-3454 on the survival rates of leukemic mice by utilizing the MOLM13 xenotransplantation model, which represents a very aggressive model of MLL leukemia in which terminal disease develops within 2 weeks after transplantation. Treatment with MI-3454 (100 mg/kg, b.i.d., p.o.) or vehicle was initiated 5 days after transplantation of mice with MOLM13 cells (harboring MLL-AF9) and was continued for 19 consecutive days, with no signs of toxicity or reduced mouse body weight (Supplemental Figure 5E). As expected, the vehicle-treated mice quickly developed terminal leukemia, with a median survival of 16 days (Figure 2F and Supplemental Figure 5, F and G). In contrast, MI-3454 very effectively blocked leukemia progression during the treatment period, as validated by an analysis of hCD $45^{+}$ cells in the peripheral blood of the mice, which demonstrated less than $0.2 \%$ of $\mathrm{hCD} 45^{+}$cells during MI-3454 treatment (Figure $2 \mathrm{G})$. A median survival of 35 days for the MI-3454-treated mice was observed, representing an approximately $120 \%$ increase in the survival rate over vehicle control, with evidence of leukemia progression only upon withdrawal of the compound. These results reveal a strong antileukemic effect of MI-3454 in the very aggressive MOLM13 model of MLL leukemia, suggesting also that continuous treatment with a menin-MLL1 inhibitor might be needed to block leukemia progression in this model.

MI-3454 impairs the clonogenic potential of AML primary patient samples with MLL1 translocations or NPM1 mutations. We next aimed to establish the activity of MI-3454 in primary leukemia samples (Supplemental Table 2). First, we tested this compound in primary AML samples with MLL1 translocations. Treatment with low-nanomolar concentrations of MI-3454 (3-12 $\mathrm{nM}$ ) substantially reduced clonogenic potential in all MLL leukemia patient samples when compared with DMSO control, which 
A

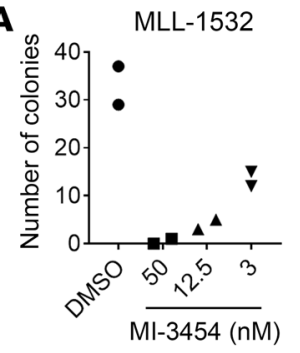

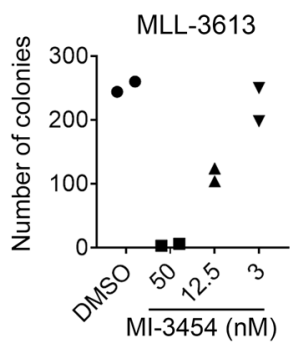
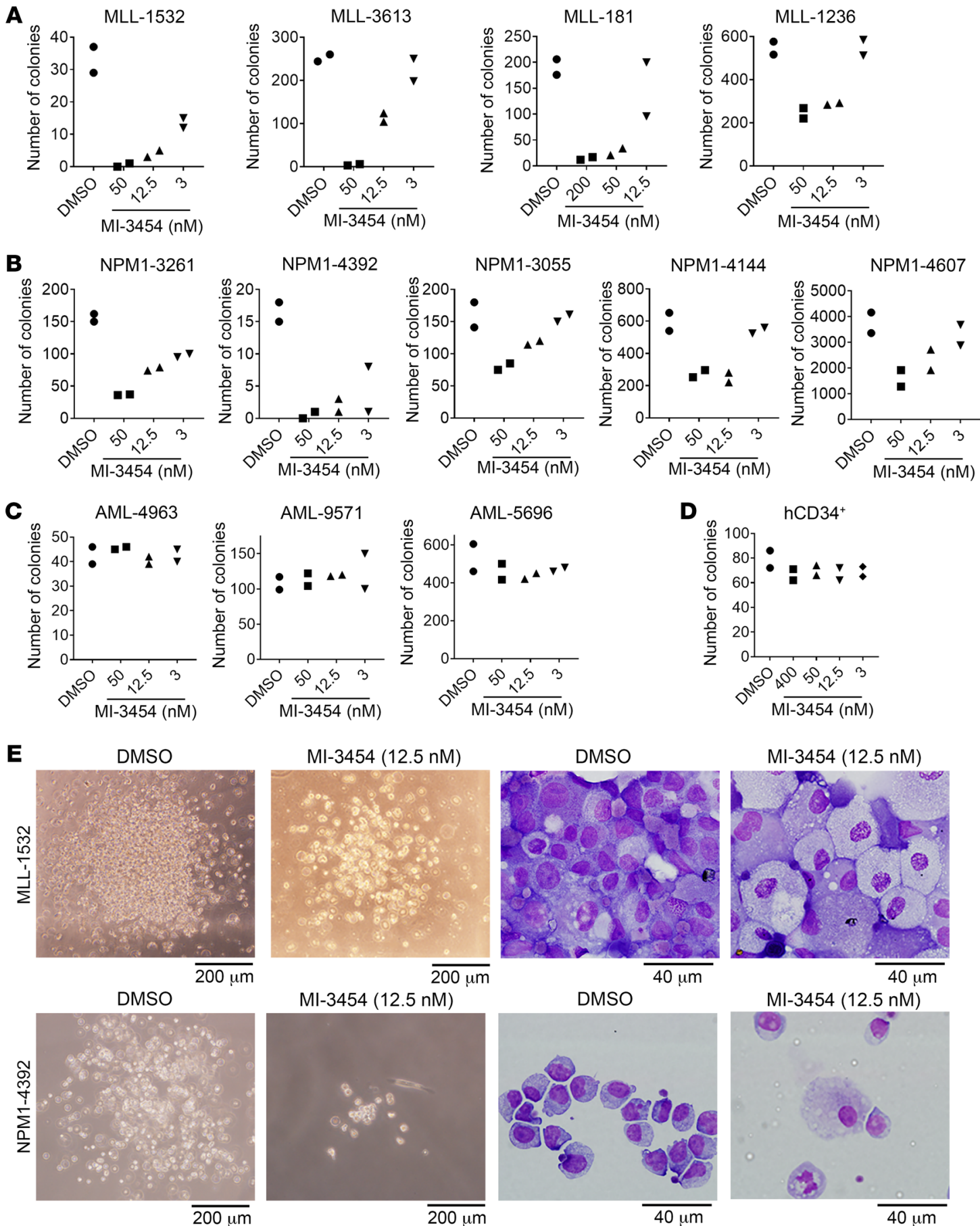

MI-3454 (12.5 nM)
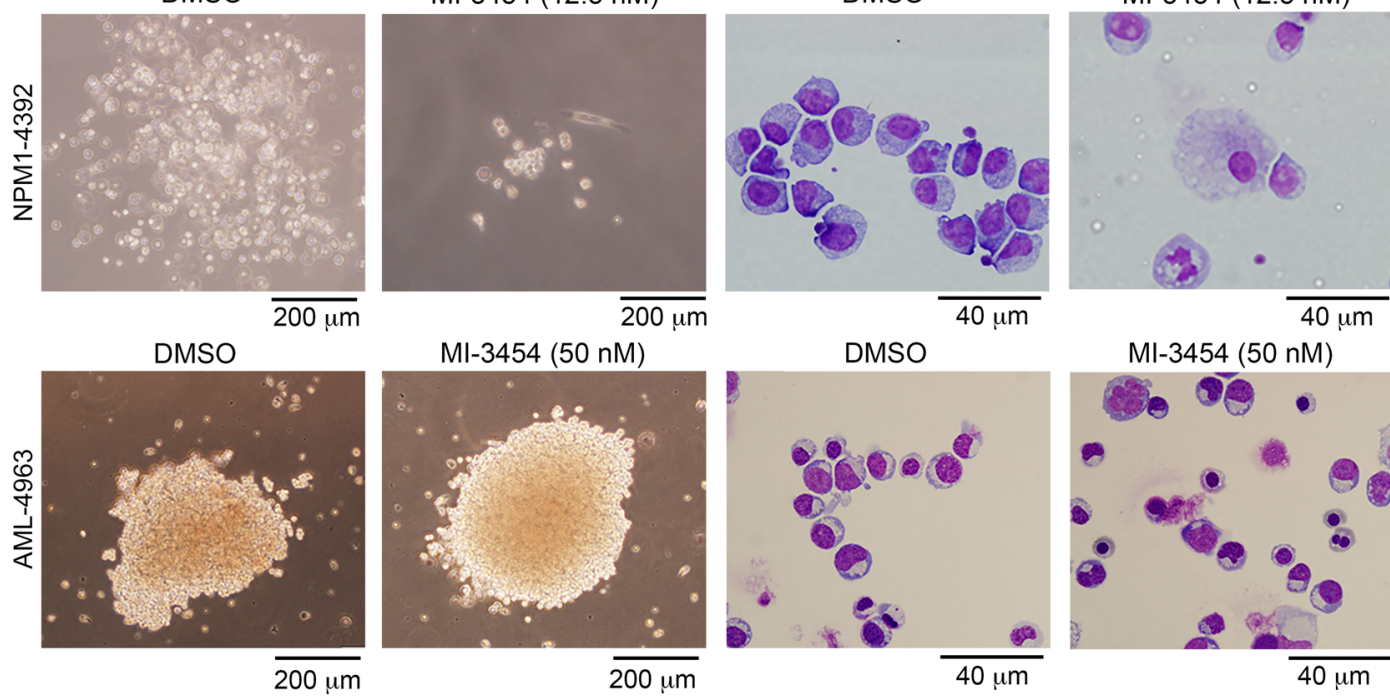

$\mathrm{Ml}-3454(50 \mathrm{nM})$

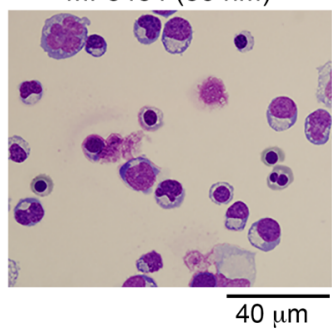

Figure 3. Activity of MI-3454 in leukemia patient samples. (A-D) Colony counts for methylcellulose colony-forming assay performed upon 7-10 days of treatment with MI-3454 in primary patient samples with MLL leukemia (A), NPM1-mutated AML samples (B), control AML primary samples (without MLL1 rearrangement or NPM1 mutations) (C), and in normal hematopoietic CD34+ cells isolated from cord blood (D). Individual data points for each sample are shown, $n=2$. (E) Images of colonies and Wright-Giemsa-stained cytospins for colony-forming assay in selected primary AML samples upon treatment with MI-3454 or DMSO. 
A $\quad$ MLL-1532 (PDX)

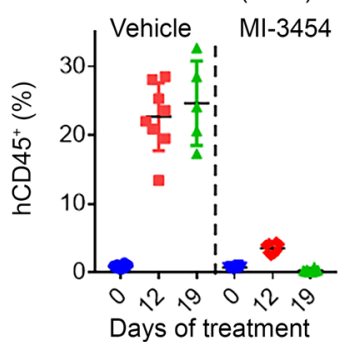

B

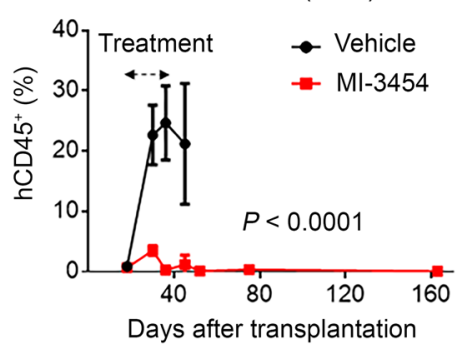

C

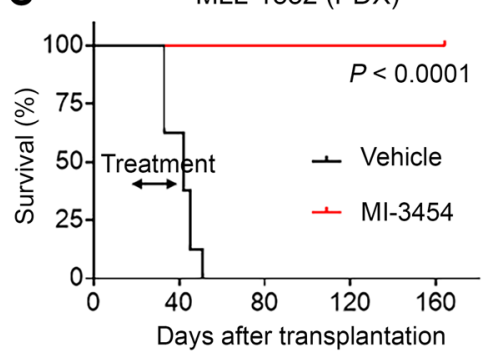

D

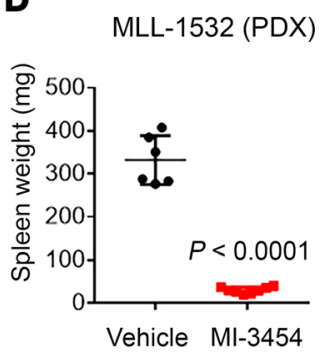

$\mathbf{E}$

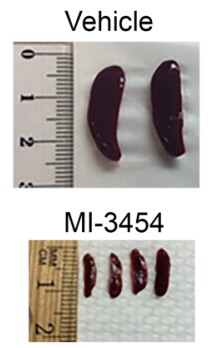

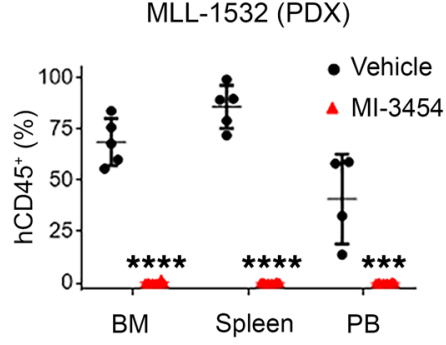

$\mathbf{F}$

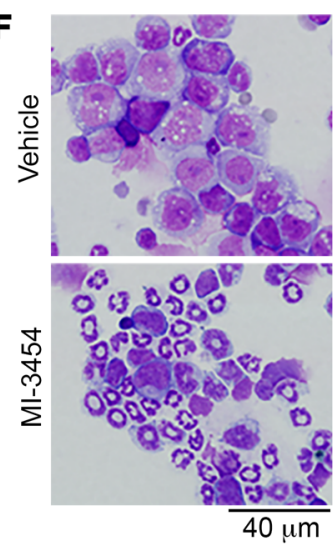

G

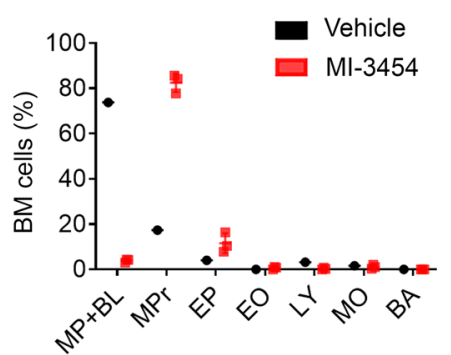

H

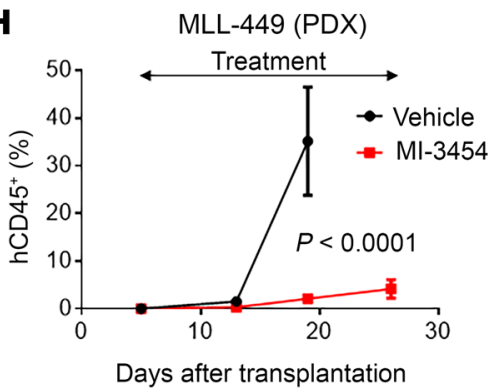

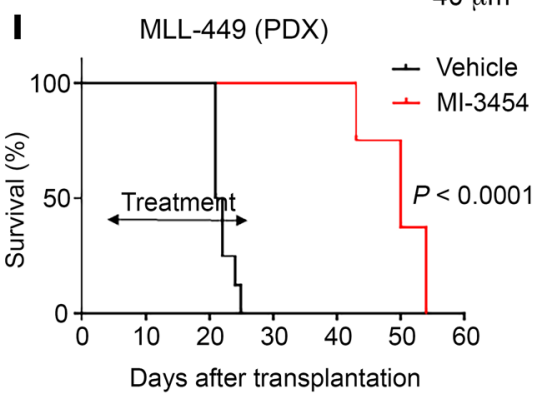

Figure 4. In vivo activity of MI-3454 in PDX models of MLL leukemia. (A and B) Flow cytometric quantification of human CD45 cells in peripheral blood (PB) of MLL-1532 PDX mice during treatment of mice with MI-3454 (100 mg/kg, b.i.d., p.o.) or vehicle (A and B) as well as after stopping the treatment (B). Mean \pm SD, $n=8$. (C) Kaplan-Meier survival curves of vehicle- or Ml-3454-treated (100 mg/kg, b.i.d., p.o.) mice in the MLL-1532 PDX model ( $n=8$ ). (D) Weight and images of spleens collected from MLL-1532 PDX mice treated with vehicle (days 33-51 after transplantation) or MI-3454 (day 164 after transplantation). Mean $\pm S D, n=6-8$. (E) Flow cytometric quantification of human CD45 cells in peripheral blood (PB), spleen, and bone marrow (BM) samples harvested from the MLL-1532 PDX mice from the MI-3454 (on day 164 after transplantation) or vehicle (at terminal stage of leukemia: days 33-51 after transplantation) cohort. Mean $\pm \mathrm{SD}, n=5$ (vehicle) and $n=8$ (MI-3454). ${ }^{* *} P<0.001,{ }^{* * *} P<0.0001$. (F) Wright-Ciemsa-stained cytospins for bone marrow samples isolated from the MLL-1532 PDX mice. (C) Differential counting of cells in bone marrow cytospins presented in $\mathbf{F} ; n=1$ (vehicle) and $n=$ 3 (MI-3454). MP+BL, murine progenitors plus leukemic blasts; MPr, myeloid precursors, including neutrophils; EP, erythroid precursors; EO, eosinophils; LY, lymphocytes; MO, monocytes; BA, basophils. (H) Flow cytometric quantification of human CD45 cells in peripheral blood of MLL-449 PDX mice upon treatment with MI-3454 (100 mg/kg, b.i.d., p.o.) or vehicle. Mean \pm SD, $n=8$. (I) Kaplan-Meier survival curves of vehicle- or MI-3454-treated (100 mg/kg, b.i.d., p.o.) mice in the MLL-449 PDX model $(n=8)$. P values were calculated using 2-way ANOVA with Sidak's multiple-comparisons test (B and $\mathbf{H})$, logrank (Mantel-Cox) test (C and I), and Student's 2-tailed $t$ test (D and $\mathbf{E})$.

was reflected by a marked decrease in colony number and size (Figure 3A and Supplemental Figure 6A). Furthermore, MI-3454 induced morphological changes in primary cells with $M L L 1$ translocations, suggesting differentiation of these cells from leukemic blasts to more mature hematopoietic cells (Figure 3E and Supplemental Figure 6A).

Previous studies have demonstrated that inhibition of the menin interaction with a wild-type MLL1 blocks the proliferation of AML cells with NPM1 mutations (24). Therefore, we tested the activity of MI-3454 in NPM1-mutated AML patient samples and found a marked reduction in colony-forming ability upon treatment with low-nanomolar concentrations of MI-3454, as reflected by decreased colony number and reduced colony size and differentiated phenotype of cells (Figure 3, B and E, and Supplemental Figure 6B). Importantly, the effect of MI-3454 in the NPM1mutated AML samples did not seem to be dependent on the type of secondary mutations (e.g., FLT3-ITD in samples 3261 and 3055, DNMT3A $A^{\text {mut }}$ and TET2 $2^{\text {mut }}$ in sample 4392, IDH2 ${ }^{\text {mut }}$ in sample 4144, and TET2 ${ }^{\text {mut }}$ in sample 4607; Supplemental Table 2), as all these samples were sensitive to the treatment with MI-3454. 


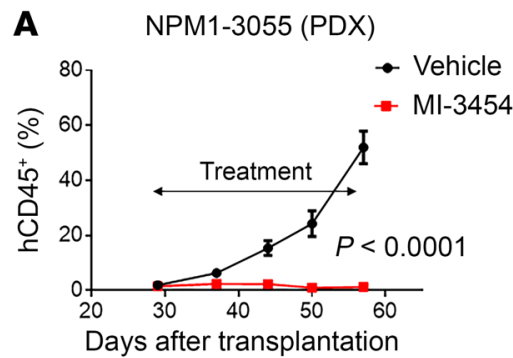

D
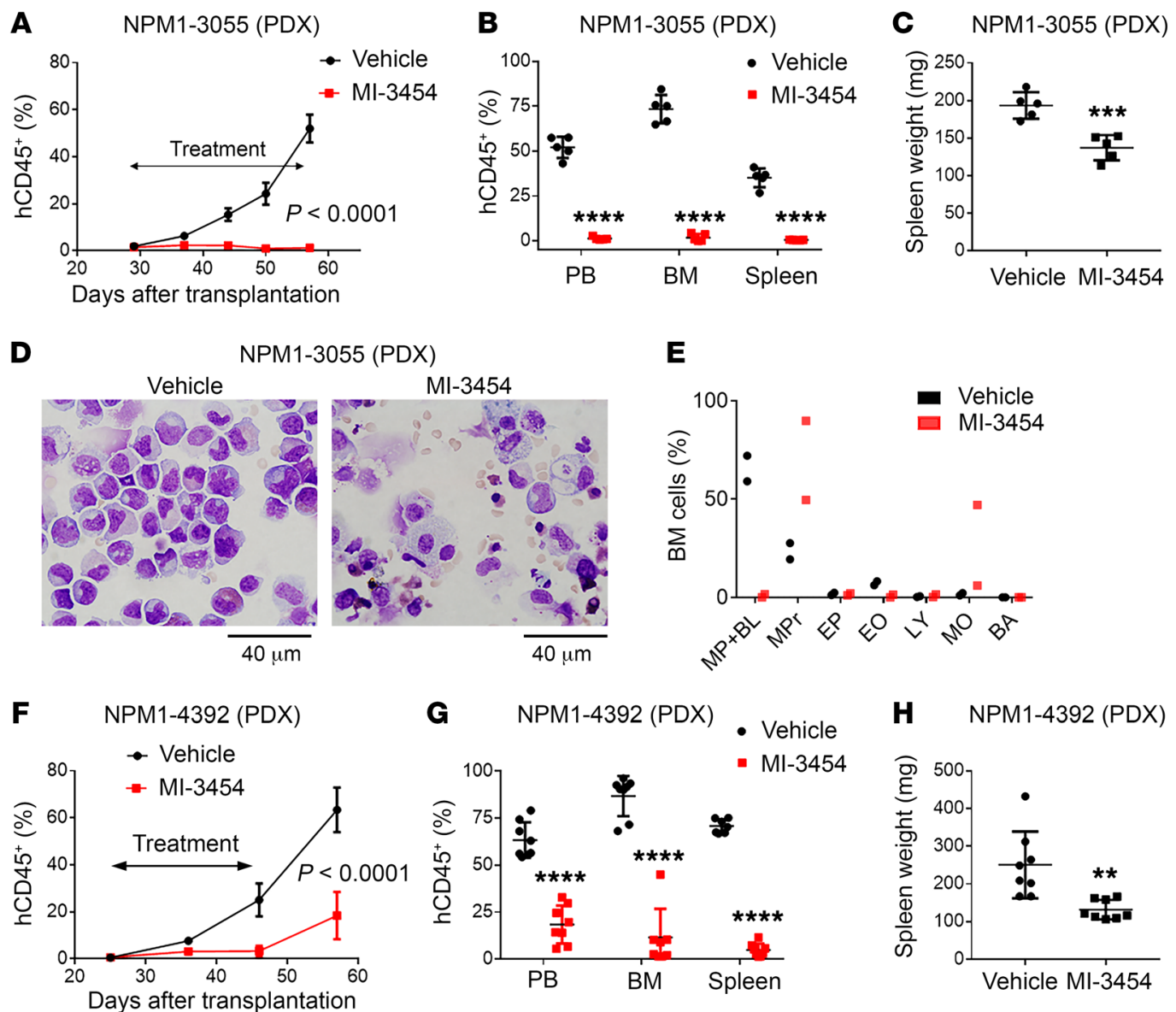

I

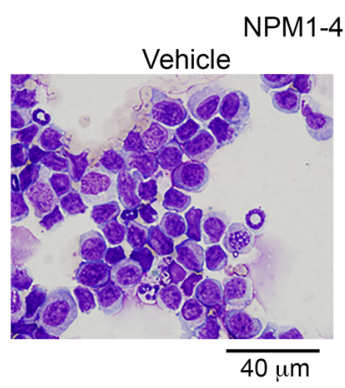

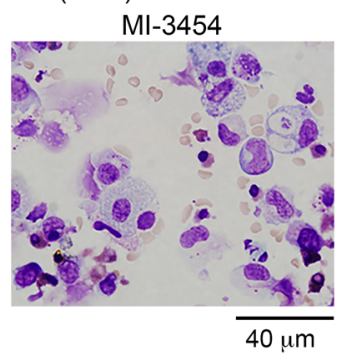

E

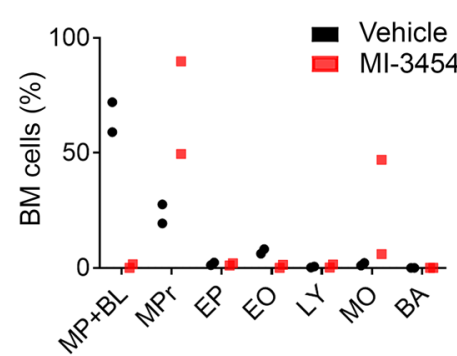

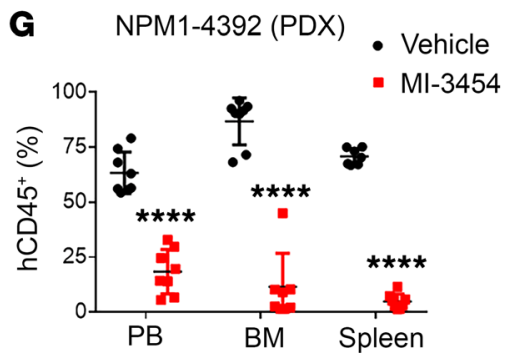

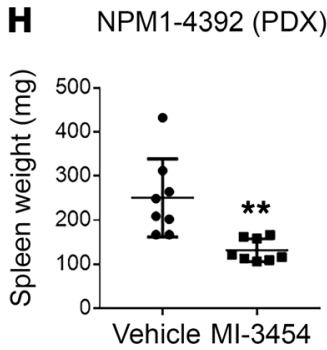

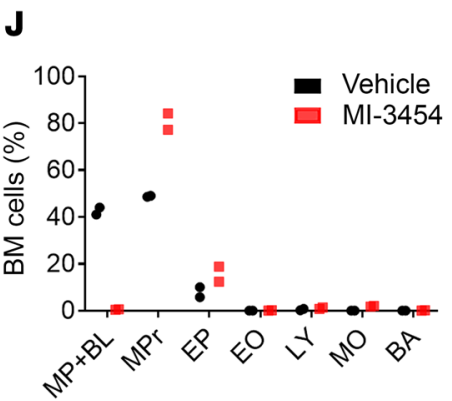

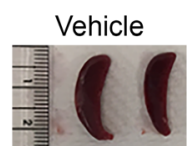

$\mathrm{Ml}-3454$

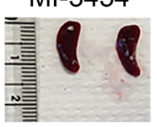

Figure 5. In vivo activity of MI-3454 in PDX models of NPM1-mutated AML. (A) Flow cytometric quantification of human CD45+ cells in peripheral blood of NPM1-3055 PDX mice during treatment of mice with MI-3454 (100 mg $/ \mathrm{kg}$, b.i.d., p.o.) or vehicle. Mean \pm SD, $n=5$ per group. (B) Flow cytometric quantification of human $\mathrm{CD}_{4} 5^{+}$cells in peripheral blood (PB), spleen, and bone marrow (BM) harvested at the endpoint of the experiment from the NPM1-3055 PDX mice. Mean \pm SD, $n=5$. (C) Spleen weight of NPM1-3055 PDX mice collected at the endpoint of the experiment. Mean \pm SD, $n=5$. (D) Wright-Ciemsastained cytospins for bone marrow samples isolated from the NPM1-3055 PDX mice upon treatment with MI-3454 or vehicle. (E) Differential counting of cells in bone marrow cytospins presented in $\mathbf{D} ; n=2$. Labeling of cell types as in Figure 4G. (F) Flow cytometric quantification of human CD45+ cells in peripheral blood of NPM1-4392 PDX mice upon treatment with MI-3454 (100 mg/kg, b.i.d., p.o.) or vehicle. Mean \pm SD, $n=8$. (C) Flow cytometric quantification of human $\mathrm{CD}_{4} 5^{+}$cells in peripheral blood, spleen, and bone marrow samples harvested at the endpoint of the experiment from the NPM1-4392 PDX mice. Mean \pm SD, $n=8$. (H) Spleen weight and images of spleens from NPM1-4392 PDX mice treated with MI-3454 or vehicle collected at the endpoint. Mean \pm SD, $n=8$. (I) Wright-Giemsa-stained cytospins for bone marrow samples isolated from the NPM1-4392 PDX mice. (J) Differential counting of cells in bone marrow cytospins presented in I; $n=2$ per cohort. Cell labeling as in Figure 4G. In B, C, G, and $\mathbf{H},{ }^{* *} P<0.01 ;{ }^{* *} P<0.001 ;{ }^{* * * *} P<0.0001$ calculated using Student's 2-tailed $t$ test. In $\mathbf{A}$ and $\mathbf{F}, P$ values were calculated using 2-way ANOVA with Sidak's multiple-comparisons test.

We also assessed the effect of MI-3454 on colony formation in primary AML samples without MLL1 translocations or NPM1 mutations, such as sample 9571 (harboring FLT3-ITD and IDH mutations), 4963 (with FLT3, U2AF1, ZRSR2, and WT1 mutations), and 5696 (with FLT3-ITD, WT1, and ASXL1 mutations), which are not expected to be dependent on the menin-MLL1 interaction. Indeed, we did not observe any effect of MI-3454 on colony formation in these samples (Figure 3, C and E, and Supplemental Figure 6C). We also tested the activity of MI-3454 in normal hematopoietic progenitor cells (human $\mathrm{CD} 34^{+}$cord blood cells) and observed no effect on colony formation 

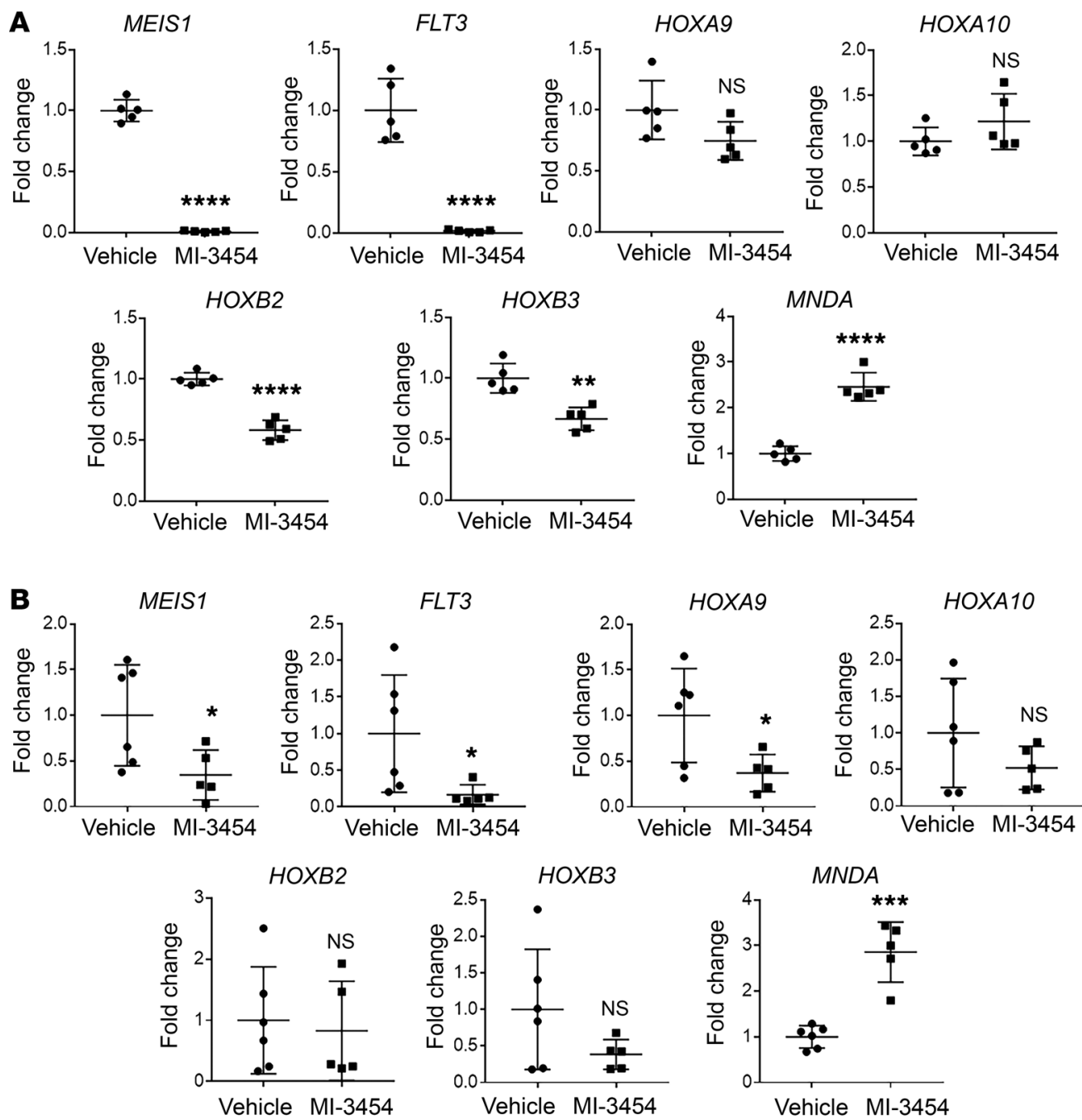

Figure 6. Gene expression changes induced by MI-3454 in the NPM1-mutated PDX models.

(A) Gene expression measured by qRT-PCR of RNA extracted from bone marrow samples in the NPM13055 PDX model harvested at the endpoint of the experiment (day 57 after transplantation) after 26 days of mice treatment with MI-3454 (100 mg/kg, b.i.d., p.o.) or vehicle. TaqMan primers and probes specific for human genes were used. Transcripts levels are normalized to $G A P D H$ and referenced to the mean transcript level in the vehicle-treated group, which is set as 1 ( $n=5$ independent samples per group). Error bars represent SD. (B) Gene expression measured by qRT-PCR of RNA extracted from bone marrow samples in the NPM1-4392 PDX model. Samples were harvested at the endpoint of the experiment (day 57 after transplantation) after 21 days of treatment of mice with MI-3454 (100 mg/kg, b.i.d., p.o.) or vehicle. TaqMan primers and probes specific for human genes were used. Transcript levels are normalized to $G A P D H$ and referenced to the mean transcript level in the vehicle-treated group, which is set as 1 ( $n=5-6$ independent samples per group). Error bars represent $\mathrm{SD}$. In $\mathbf{A}$ and $\mathbf{B},{ }^{*} P<0.05 ;{ }^{* *} P<0.01$ ${ }^{* * *} P<0.001 ;{ }^{* * *} P<0.0001$; NS, not significant; calculated using Student's 2-tailed $t$ test. at all concentrations tested (Figure 3D). Taken together, these results show potent and selective activity of MI-3454 in primary AML samples with $M L L 1$ translocations or NPM1 mutations but not in other subtypes of leukemia or normal hematopoietic progenitor cells.

MI-3454 induces complete remission or blocks leukemia progression in PDX models of MLL leukemia. The PDX models utilizing human primary tumor samples represent highly clinically relevant preclinical models, as they maintain the genetic profiles and histopathological features of the original patient tumors. Thus, we aimed to assess the in vivo efficacy of MI-3454 in PDX models derived from leukemia patient samples harboring $M L L 1$ translocations that were obtained from both adult and infant patients. First, we developed a PDX model derived from sample 1532, which harbors the MLL-p300 fusion protein and was obtained from an adult MLL leukemia patient (Supplemental Figure 7A). In the efficacy experiment utilizing the MLL-1532 PDX model, the engraftment of leukemic cells was validated 19 days after transplantation, demonstrating approximately $20 \%$ leukemic blasts in bone marrow (Supplemental Figure 7B). At that time point, treatment with MI-3454 (100 mg/kg, b.i.d., p.o.) or vehicle was initiated and continued for 20 consecutive days, without affecting mouse body weight or other signs of toxicity (Supplemental Figure 7C). Leuke- mia developed rapidly in the vehicle-treated mice, as manifested by a substantial increase in the level of leukemic blasts (hCD $45^{+}$ cells) (Figure 4A). All vehicle-treated mice developed terminal leukemia, with 42-day median survival, as reflected by a high level of leukemic blasts in the bone marrow, peripheral blood, and spleen, and enlarged spleen size (Figure 4, B-F). Interestingly, mice treated with MI-3454 showed an initial increase in the level of hCD $45^{+}$cells in the peripheral blood (on day 12 of treatment), which then dropped to a very low level (below 0.5\%) on day 19 of treatment (Figure 4, A and B, and Supplemental Figure 7D), consistent with regression of leukemia.

Remarkably, no signs of leukemia were observed in any of the MLL-1532 PDX mice in the MI-3454 cohort even several months after discontinuing the treatment (e.g., on day 164 after transplantation), as reflected by an undetectable level of hCD $45^{+}$cells in the peripheral blood (Figure 4, B and C) and no external signs of leukemia. At that time, the MI-3454-treated mice were euthanized and flow cytometric analysis of the bone marrow, peripheral blood, and spleen samples was performed, demonstrating the absence of leukemic blasts $\left(<0.1 \%\right.$ of $\mathrm{hCD} 45^{+}$cells in these samples) (Figure 4E and Supplemental Figure 7E). Furthermore, the average spleen size in the MI-3454 cohort of mice was substan- 
A

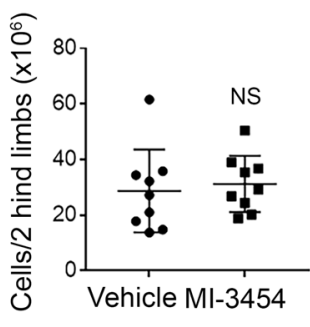

B

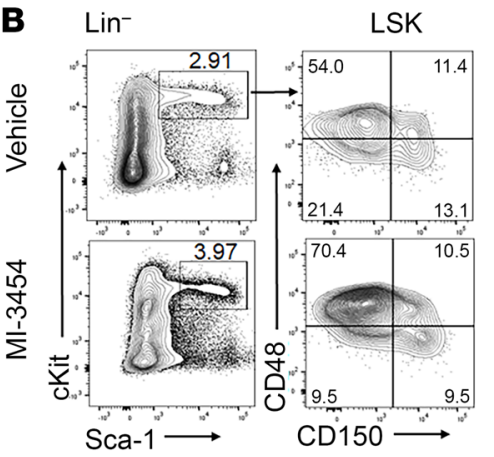

C
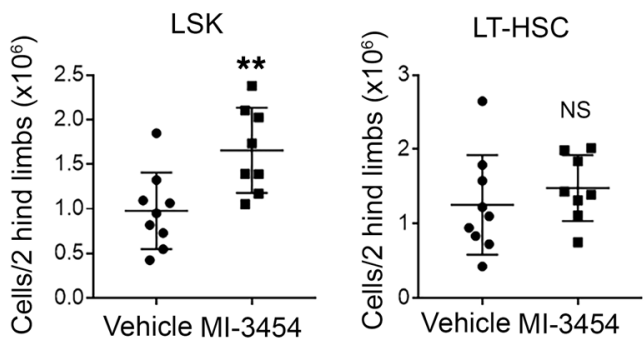

E

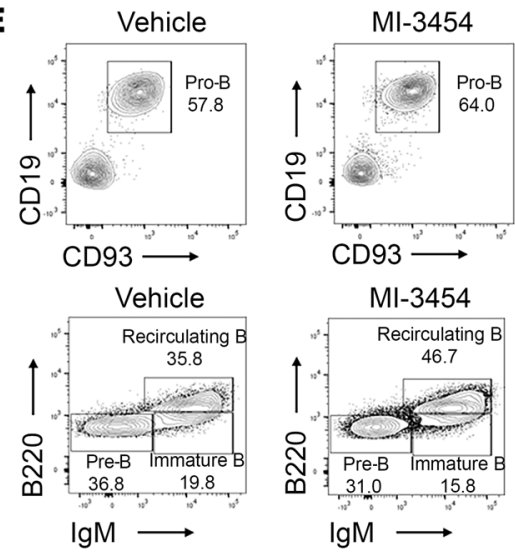

D

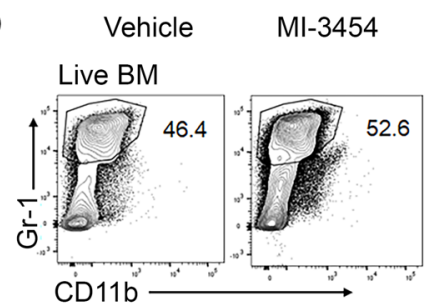

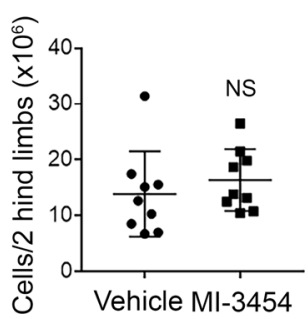

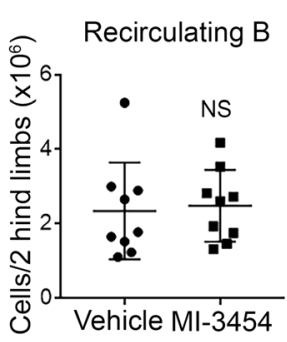

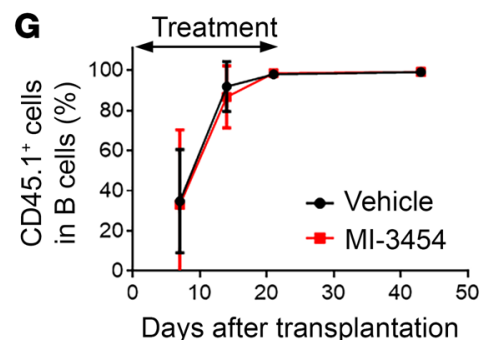

Days after transplantation

H

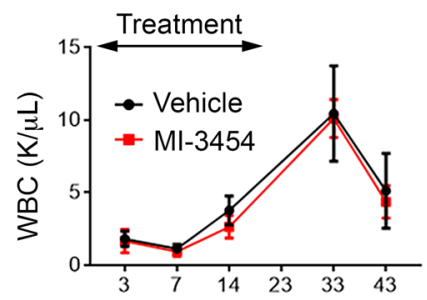

Days after transplantation
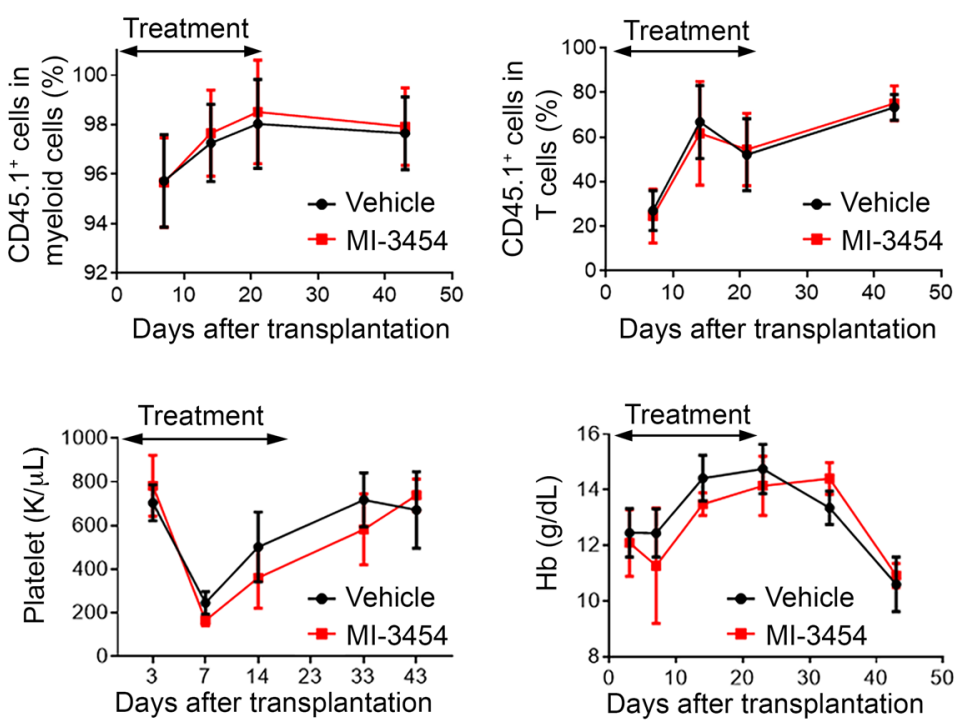

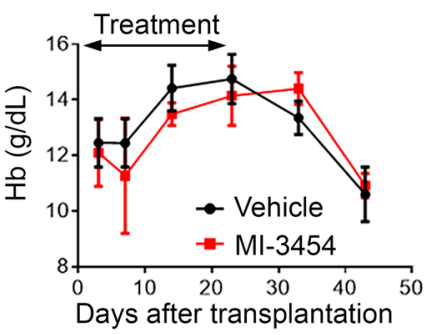


Figure 7. MI-3454 does not impair normal hematopoiesis. (A) Analysis of bone marrow cellularity after 19 days of treatment of normal C57BL/ 6 mice with MI-3454 (100 mg/kg, b.i.d., p.o.) or vehicle ( $n=9 /$ group, mean \pm SD). NS, not significant. (B) Representative contour plots from flow cytometric analysis of hematopoietic stem and progenitor cells: LSK (Lin-Sca-1+c-Kit $\left.{ }^{+}\right)$ and long-term hematopoietic stem cells (LT-HSCs, defined as Lin-Sca-1+ C-Kit+CD48-CD150+). (C) Quantification of LSK and LT-HSC cell frequency by flow cytometry ( $n=8-9 /$ group, mean $\pm \mathrm{SD}$ ). ${ }^{* *} P<0.01$ (treatment is described in A). (D) Representative contour plots (left panel) and quantification (right panel) from flow cytometric analysis of myeloid cells in bone marrow (BM) samples isolated from mice treated with vehicle or MI-3454 ( $n=8-9 /$ group, mean \pm SD); treatment is described in $\mathbf{A}$. ( $\mathbf{E}$ and $\mathbf{F}$ ) Representative contour plots from flow cytometric analysis $(\mathbf{E})$ and quantification of absolute numbers (F) of B cell populations in bone marrow $(n=9$ / group, mean $\pm \mathrm{SD}$ ). Contour plots in $\mathbf{E}$ were pregated on $\mathrm{B} 22 \mathrm{O}^{+} \mathrm{CD} 43^{+} \mathrm{s} / \mathrm{gM}$ cells (pro-B cells) and $B 220^{+} C D 43^{-}$cells (pre-B, immature $B$, recirculating $B$ cells). In A, C, D, and F, ${ }^{* *} P<0.01$; NS, not significant; calculated using unpaired Welch's $t$ test. (C) Quantification of the population of donor cells (CD45.1+) within the specific subtype of blood cells in the C57BL/6-CD45.2 recipient mice after bone marrow transplantation and upon 21 days of treatment with vehicle or Ml-3454 (100 mg/ kg, b.i.d., p.o.). Mean \pm SD, $n=7-8$. (H) Blood parameters: white blood cells (WBC), platelets, and hemoglobin $(\mathrm{Hb})$ in C57BL/6-CD45.2 recipient mice after bone marrow transplantation and upon 21 days of treatment with vehicle or MI-3454. Mean \pm SD, $n=7-8$. K, thousand. Differences in $\mathbf{G}$ and $\mathbf{H}$ are not significant between the 2 cohorts as calculated using 2-way ANOVA with Sidak's multiple-comparisons test.

tially smaller when compared with the vehicle control mice, and bone marrow cells demonstrated a highly differentiated phenotype in the MI-3454-treated mice (Figure 4, D, F, and G). In addition, in contrast with the vehicle-treated mice, we did not observe tissue infiltration with leukemic blasts in the mice treated with MI-3454 (Supplemental Figure 7F). These results demonstrate a complete eradication of leukemic blasts by MI-3454, providing evidence that the treatment cured mice of MLL leukemia in this PDX model. To assess whether MI-3454 affects the frequency of leukemia-initiating cells in primary sample 1532, we treated ex vivo $\mathrm{CD} 4^{+}$cells isolated from primary sample 1532 with MI-3454 or DMSO and found that low-nanomolar concentrations of MI-3454 can markedly reduce the level of hCD34 ${ }^{+}$cells (Supplemental Figure 7, G and H). These results suggest that MI-3454 might be capable of reducing leukemia initiating cells, although additional studies in a broader panel of primary leukemia samples are required to further validate these findings.

Subsequently, we developed a PDX model of pediatric leukemia derived from an infant B cell ALL (B-ALL) patient (sample 449) that expressed MLL-ENL fusion protein. This PDX represents a very aggressive MLL leukemia model, with a median mouse survival reaching 23 days (Supplemental Figure 8, A and B). Five days after transplantation of NSGS mice with sample 449, treatment with MI-3454 (100 mg/kg, p.o., b.i.d.) or the vehicle was initiated and continued for 21 consecutive days, with no negative effects on body weight in the MI-3454 cohort (Supplemental Figure 8C). Notably, a substantial reduction in leukemia progression was observed in the MI-3454 cohort versus vehicle-treated mice in this PDX model, as reflected by a markedly reduced level of hCD $45^{+}$cells in the peripheral blood (Figure 4H and Supplemental Figure 8, D and E). Although all control mice developed terminal leukemia within approximately
21 days, the MI-3454-treated group had substantially prolonged survival and reached a median survival of 50 days, indicating an approximately $140 \%$ increase in the duration of survival over the vehicle-treated mice (Figure 4I). Even though MI-3454 did not completely eradicate leukemic cells in this aggressive PDX model of infant B-ALL, a very profound in vivo effect of this compound was observed. Overall, our results strongly support the therapeutic potential of MI-3454 in both adult and pediatric leukemias with $M L L 1$ translocations.

MI-3454 blocks leukemia progression in PDX models of AML with NPM1 mutations. Because MI-3454 substantially reduced colony formation in AML patient samples with NPM1 mutations, we aimed to test the activity of this compound in the in vivo PDX models of the NPM1-mutated leukemia. We developed 2 PDX models from AML samples with NPM1 mutations: 3055 and 4392 (Figure 3B and Supplemental Table 2), which were successfully engrafted in NSGS mice. In both models, we initiated treatment with MI-3454 (100 mg/kg, p.o., b.i.d.) or vehicle on day $25-28$ after transplantation and continued treatment for 21 consecutive days. Because of the relatively long latency time required for leukemia development in control mice in these PDX models (over 50 days), treatment efficacy was assessed by monitoring the level of engraftment of leukemic blasts (hCD $45^{+}$cells) in these mice.

In the PDX model derived from sample 3055 (with NPM1 mutation and FLT3-ITD), the level of leukemic blasts in the peripheral blood increased substantially in the vehicle-treated mice (to $55 \%$ on day 56 after transplantation) (Figure $5 \mathrm{~A}$ ). In contrast, treatment with MI-3454 greatly reduced the level of leukemic blasts below that observed when the treatment was initiated (Figure 5A), suggesting leukemia regression. After completing the treatment (on day 56 after transplantation), all mice were euthanized, and very high levels of leukemic blasts were detected in all samples isolated from the vehicle-treated mice (e.g., $>70 \%$ hCD $45^{+}$cells in the bone marrow samples), with marked reduction in the MI-3454treated mice (e.g., $<3 \%$ in bone marrow samples) (Figure $5 \mathrm{~B}$ and Supplemental Figure 9A). These effects were accompanied by significantly lower spleen weights, a differentiating phenotype of bone marrow cells (Figure 5, C-E), a reduced white blood cell count without a negative effect on other blood parameters (Supplemental Figure 10, A-D), and a lack of infiltration of leukemic blasts in different organs in the MI-3454-treated versus vehicle control mice (Supplemental Figure 9B).

Similarly, in the PDX model derived from the primary leukemia sample 4392, which harbored NPM1, DNMT3A, and TET2 mutations, the level of $\mathrm{hCD} 45^{+}$cells in the peripheral blood of the mice remained very low and steady over the course of treatment with MI-3454, while it increased substantially in the vehicle control mice (Figure 5F). Flow cytometric analysis of the samples isolated at the endpoint of the experiment (day 56 after transplantation) demonstrated substantially reduced levels of leukemic blasts in the peripheral blood, bone marrow, and spleen samples following treatment with MI-3454 versus vehicle control (Figure 5G and Supplemental Figure 9C). Furthermore, significantly smaller spleen size (Figure $5 \mathrm{H}$ ), highly differentiated phenotype of bone marrow cells (Figure 5, I and J), and markedly reduced blast infiltration of organs (liver and bone marrow) following treatment with MI-3454 was observed (Supplemental Figure 9D), indicating a sig- 
nificant reduction in leukemia progression induced by MI-3454. Of note, stopping the treatment with MI-3454 led to an increase in the level of leukemic blasts (Figure 5F), suggesting that longer or continued treatment with MI-3454 might be required to fully suppress leukemia progression in this PDX model. Taken together, these data demonstrate that inhibition of the menin-MLL1 interaction by MI-3454 very effectively blocks leukemia progression in clinically relevant PDX models of AML with NPM1 mutations.

Treatment with MI-3454 markedly downregulates MEIS1 and FLT3 expression in NPM1-mutated AML. To investigate the mechanism of action of MI-3454 in the PDX models of NPM1-mutated AMLs, we performed gene expression studies using bone marrow or spleen samples isolated from mice treated with vehicle or MI-3454. Previous studies suggested that the menin-MLL1 interaction is involved in the regulation of HOXA9, HOXA1O, and $H O X B$ cluster genes and MEIS1, which play an important role in the NPM1-mutated leukemias $(24,26-28,35)$. Interestingly, gene expression studies that we performed in the bone marrow samples isolated from the sample 3055 PDX mice (harboring NPM1 and FLT3-ITD mutations) demonstrated no significant reduction in HOXA9 or HOXA1O expression upon mouse treatment with MI-3454 (Figure 6A). In contrast, we observed profound downregulation of MEIS1 and FLT3 (by 100- and 65-fold, respectively) in samples isolated from mice treated with MI-3454 (Figure $6 \mathrm{~A})$. Furthermore, the expression of $\mathrm{HOXB}$ cluster genes, such as HOXB2 and HOXB3, was downregulated in the MI-3454 cohort of mice, although to a much lower extent than MEIS1 or FLT3 (Figure $6 \mathrm{~A})$. These effects were accompanied by a significant increase in the expression level of the MNDA differentiation marker (Figure $6 \mathrm{~A})$, consistent with the differentiating phenotype of bone marrow cells isolated from these PDX mice (Figure 5D).

We also performed gene expression studies in spleen samples isolated at the endpoint of the experiment from the sample 4392 PDX mice (with NPM1, DNMT3A, and TET2 mutations) treated with MI-3454 or vehicle. In this model, the most profound downregulation ( 6-fold) was observed for FLT3 expression, while MEIS1 and HOXA9 were affected to a lower extent ( 2.5 -fold) in the MI-3454-treated mice when compared with the vehicle control mice (Figure 6B). Furthermore, the HOXA1O and HOXB cluster genes (e.g., $H O X B 2$ and $H O X B 3$ ) were not affected significantly, while the $M N D A$ differentiation gene was markedly upregulated in the samples isolated from the MI-3454-treated sample 4392 PDX mice (Figure 6B). To further validate these findings, we performed in vitro gene expression studies using NPM1-mutated AML sample 5046 (with NPM1, DNMT3A, TET2, and FLT3 mutations), which was obtained from the same patient as sample 4392 after relapse. Of note, the 4392 primary sample could not be used in these studies due to the lack of additional available material. Importantly, very similar gene expression changes were observed in the primary AML sample 5046 as in the sample 4392 PDX, with MEIS1 and FLT3 showing the most significant reduction upon treatment with MI-3454 (5- to 10-fold), while HOXA9 and HOXA1O were reduced to a lower extent (up to 3-fold) (Supplemental Figure 11). Overall, our gene expression studies suggest that MEIS1 and FLT3 are the key genes associated with the inhibition of the menin-MLL1 interaction in the NPM1-mutated AML PDX models or primary samples regardless of secondary mutations present. These results support the concept that MEIS1 could serve as a biomarker of treatment efficacy with menin-MLL1 inhibitors in the NPM1-mutated AMLs in a clinical setting.

MI-3454 does not affect normal hematopoiesis and is not toxic to normal mice. Because MLL1 plays a role in normal hematopoiesis $(36,37)$, we assessed whether the menin-MLL1 inhibitor MI-3454 affects normal hematopoiesis in mice. We treated C57BL/6 mice for 19 consecutive days with vehicle or MI-3454 using the same treatment regimen applied in the PDX models of AML $(100 \mathrm{mg} /$ $\mathrm{kg}$, p.o., b.i.d.). Importantly, no decrease in body weight or tissue damage was found following treatment with MI-3454 (Supplemental Figure 12, A and B). We then analyzed bone marrow samples isolated from the mice and found that bone marrow cellularity was not affected by MI-3454 (Figure 7A). Flow cytometric analysis revealed that the relative and absolute numbers of phenotypically defined hematopoietic stem and progenitor cells were not decreased upon treatment with MI-3454 (Figure 7, B and C) (LSK population defined as $\mathrm{Lin}^{-} \mathrm{Sca}-1^{+} \mathrm{c}-\mathrm{Kit}^{+}$and long-term hematopoietic stem cells, LT-HSCs, defined as LSK CD $48^{-} \mathrm{CD}^{-}{ }^{+} \mathrm{O}^{+}$). In fact, the abundance of LSK cells in the bone marrow was slightly increased in the MI-3454-treated cohort of mice over vehicle control mice (Figure 7C) as well as the overall Sca-1 expression. Furthermore, an analysis of bone marrow myeloid cells demonstrated no significant impact of MI-3454 on the relative or absolute number of these cells (Figure 7D). A phenotypic analysis of B cell populations (pro- $\mathrm{B}$, pre- $\mathrm{B}$, immature $\mathrm{B}$, and recirculating $\mathrm{B}$ cells) revealed no significant impact on any of the $B$ cell populations in the MI-3454-treated mice as compared to the vehicle control mice (Figure 7, E and F). These results are consistent with studies suggesting that menin is not an essential cofactor of MLL1 during normal hematopoiesis (38-40).

In addition, we observed no significant effects on complete blood counts apart from a slight increase in platelet levels (although it remained within the normal range for both cohorts of mice) and no impact on liver enzymes in the MI-3454-treated versus vehicle control mice (Supplemental Figure 12, C and D). Finally, a histopathological analysis demonstrated no tissue damage or other abnormalities upon treatment of mice with MI-3454 (Supplemental Figure 12B). Taken together, these results demonstrate that MI-3454 preserved the number of normal hematopoietic stem and progenitor cells in mice and lacks general toxicity, indicating that a sufficient therapeutic window exists for this meninMLL1 inhibitor.

To address whether menin-MLL1 inhibition affects normal hematopoiesis in the context of hematopoietic stress, we tested the effect of MI-3454 in C57BL/6-CD45.2 mice after 5-fluorouracil (5-FU) treatment or after transplantation of bone marrow cells from B6-CD45.1 donor mice into lethally irradiated (9 Gy) C57BL/6-CD45.2 recipient mice. In both experiments, treatment with MI-3454 (100 mg/kg, p.o., b.i.d.) or vehicle was initiated on the day following 5-FU treatment or transplantation, and it was continued for 21 consecutive days. Mice were monitored for an additional 20 days after completing the treatment. Importantly, analysis of peripheral blood samples during the period of treatment did not show any negative effect of MI-3454 on the level of CD45.1 $1^{+}$donor-derived B cells, T cells, or myeloid cells as well as on complete blood counts in the recipient mice transplanted with 
donor bone marrow cells (Figure 7, G and H). Furthermore, no effect on bone marrow cellularity, LSK, LT-HSC, myeloid, and B cell populations was found at the endpoint of the experiment (20 days after completing the treatment) (Supplemental Figure 13A). Similarly, analysis of blood or bone marrow samples in 5-FU-treated mice did not reveal any significant differences between mice treated with MI-3454 or vehicle (Supplemental Figure 13, B and C). Taken together, these results demonstrate that MI-3454 does not impair hematopoietic reconstitution after transplantation or during hematopoietic recovery after 5-FU treatment, further supporting a sufficient therapeutic window for the menin-MLL1 inhibitor MI-3454.

\section{Discussion}

Acute leukemia patients with translocations of the $M L L 1$ gene have a very poor prognosis ( $<35 \% 5$-year survival), emphasizing the urgent need for the development of new, effective, and less toxic therapies. High occurrence of $M L L 1$ translocations in infant ALLs ( 80\%), which poorly respond to available treatments (9, $10)$, further supports a critical need for new therapies for these patients. The interaction of menin with MLL1 or MLL fusion proteins plays a critical role in MLL leukemia $(15,17,41)$. The meninMLL1 interaction has also been shown to play an important role in AMLs with mutations in the NPM1 gene (24). Thus, blocking the menin-MLL1 interaction with small molecules might represent a new therapeutic approach for acute leukemia patients with $M L L$ rearrangements or NPM1 mutations. Our previous work has resulted in the development of first-in-class small-molecule menin-MLL1 inhibitors with in vitro and in vivo activity in MLL leukemia models $(17,21,22)$, but these compounds required further optimization of potency and other drug-like properties before their clinical translation.

Here, we report the development of MI-3454, a subnanomolar inhibitor of the menin-MLL1 interaction, representing a second generation of the menin-MLL1 inhibitors with greatly increased potency and optimized drug-like properties. MI-3454 blocks the proliferation of the MLL1-rearranged leukemic cells at low-nanomolar concentrations, induces differentiation, and potently downregulates MLL fusion target genes, demonstrating an on-target mechanism of action and over 100-fold selectivity toward MLL1-rearranged leukemic cells. MI-3454 is also very effective in reducing the clonogenic potential of both $M L L 1$-rearranged and NPM1-mutated leukemia patient samples, but not of normal hematopoietic progenitor cells. Remarkably, the treatment of mice with MI-3454 as a single agent results in complete remission or regression of leukemia in the MLL1-rearranged or NPM1-mutated leukemia models, including PDXs. These activities were accompanied by markedly reduced expression of the MLL fusion target genes, MEIS1, HOXA9, and MEF2C (42), supporting the on-target mechanism of action of MI-3454 in vivo. We also found that the expression of MEIS1 was particularly sensitive to treatment with MI-3454 in both MLL1-rearranged and NPM1-mutated leukemias, identifying MEIS1 expression as a potential pharmacodynamic biomarker for the clinical translation of menin-MLL1 inhibitors.

PDX models of leukemia utilizing human primary patient samples represent advanced preclinical models of leukemia, having a high clinical relevance. Despite ongoing efforts to develop a target- ed therapy for MLL leukemia (e.g., DOT1L or BET inhibitors; refs. $4,5,43)$, preclinical activity of these agents has not been reported in PDX models of MLL leukemia. Remarkably, our menin-MLL1 inhibitor MI-3454 was able to completely eradicate leukemic cells in a PDX model developed from an adult AML primary sample with $M L L 1$ translocation, leading to the absence of detectable human leukemic blasts even several months after discontinuing the treatment. Such robust antitumor activity has not been observed frequently for small-molecule inhibitors applied as single agents in aggressive leukemia models, such as MLL1-rearranged leukemia. MI-3454 was also very effective in suppressing leukemia in an aggressive PDX model derived from an infant ALL patient sample with MLL1 translocation as well as in PDX models derived from the NPM1-mutated AML samples, where regression or a substantial reduction of the leukemia burden was observed. Taken together, these results reveal that the menin-MLL1 inhibitor can be very effective as a single agent in the $M L L 1$-rearranged or NPM1-mutated leukemia models derived from primary patient samples.

Previous studies in AML patient samples with NPM1 mutations revealed upregulation of HOXA cluster, HOXB cluster, and MEIS1 genes, and downregulation of MEIS1 expression in these samples reduced the expansion of primary CD $34^{+}$leukemic cells $(35,44)$. Our studies revealed pronounced in vivo efficacy of menin-MLL1 inhibitor MI-3454 and substantial downregulation of MEIS1 and FLT3 expression in the PDX models or primary AML sample harboring NPM1 mutations. These results are consistent with the studies demonstrating an important role of MEIS1 and FLT3 in the NPM1-mutated AMLs (44). However, unlike the previous in vitro studies with an earlier generation of menin-MLL1 inhibitors (24), treatment with MI-3454 resulted in only modest downregulation of HOXA9 and HOXB cluster genes (e.g., HOXB2 and HOXB3) in the PDX models of NPM1-mutated AMLs. This suggests that regulation of $H O X A 9$ and $H O X B$ cluster genes in this leukemia subtype might involve other pathways besides the menin-MLL1 interaction. Instead, the in vivo efficacy of the menin-MLL1 inhibitor might be achieved via more complex mechanisms, and we hypothesize that downregulated expression of MEIS1 and FLT3 genes might contribute to the antileukemic effect of MI-3454 in the NPM1-mutated AMLs, although validation of this hypothesis might require further mechanistic studies. Importantly, a very strong downregulation of MEIS1 expression induced by MI-3454 suggests that MEIS1 might serve as a valuable readout to assess treatment efficacy of menin-MLL1 inhibitors in leukemia and could lead to the development of a pharmacodynamic biomarker for future clinical studies.

In summary, we developed the highly potent menin-MLL1 inhibitor MI-3454 with robust activity in in vitro and in vivo models of MLL1-rearranged and NPM1-mutated leukemias, and which demonstrates on-target activity and a specific mechanism of action. Notably, MI-3454, as a single agent, induces complete remission or regression in the clinically relevant PDX models of leukemia. As menin plays a critical role in all MLL leukemias despite the nature of the $M L L 1$ fusion partner $(15,22)$, our findings should have a general applicability to all MLL leukemia subtypes. Importantly, treatment with MI-3454 did not impair normal hematopoiesis in mice, supporting the lack of on-target toxicity and showing a good therapeutic window for menin-MLL1 inhi- 
bition for future clinical studies. The effect of MI-3454 on longterm repopulation and lineage differentiation of hematopoietic stem and progenitor cells remains, however, to be established. MI-3454 represents a second-generation menin-MLL1 inhibitor with optimized drug-like properties, including a favorable PK profile and high oral bioavailability, providing a strong rationale for its clinical evaluation in leukemia patients with $M L L 1$ rearrangements or NPM1 mutations. Indeed, the Investigational New Drug application for KO-539, a structurally related analog of MI-3454 with similar in vitro and in vivo antileukemic activity (https://kuraoncology.com/wp-content/uploads/2017-AACR-NCI-EORTCMenin-Formatted-Poster-2017-10-18.pdf), was recently approved by the FDA for AML patients, and clinical trials are expected to be initiated in 2019 (NCT04067336). To date, development of MI-3454 represents one of only a few examples of highly potent inhibitors targeting protein-protein interactions with substantial promise as a new anticancer therapy. Future studies will establish a comparison of the effectiveness of MI-3454 with standard chemotherapy as well as their combination in leukemia models.

\section{Methods}

Expression and purification of human menin. The expression and purification of menin was performed as described previously $(19,33)$.

Biochemical characterization of menin-MLL1 inhibitor MI-3454. Inhibition of the menin-MLL1 interaction by MI-3454 was assessed by fluorescence polarization (FP) assay using the protocol described previously (21). Details of the FP assay are provided in the Supplemental Methods.

Crystallization of menin in complex with MI-3454. For cocrystallization experiments, $2.5 \mathrm{mg} / \mathrm{mL}$ menin was incubated with MI-3454 at a 1:4 molar ratio. Crystals were obtained using the sitting drop technique at $4^{\circ} \mathrm{C}$ by applying the procedure described previously (19). Prior to data collection, crystals were transferred into a cryosolution containing 20\% PEG 550 MME and flash-frozen in liquid nitrogen.

Crystallographic data collection and structure determination. Details on crystallographic data collection and structure determination are described in the Supplemental Methods.

In vitro profiling of MI-3454. Details on liver microsomal stability studies, inhibition of human P450 enzymes, and kinetic solubility of MI-3454 are provided in the Supplemental Methods.

Viability assays. Human leukemic cell lines MV-4-11, RS4-11, K562, REH, U937 (purchased from ATCC), KOPN-8, MOLM-13, SEM, and SET2 (purchased from DSMZ), were cultured in RPMI-1640 medium (Invitrogen) with 10\%-20\% FBS and 1\% penicillin/streptomycin. SEM cells were cultured in IMDM medium (Invitrogen) with 10\% FBS and $1 \%$ penicillin/streptomycin. For viability assay, cells were plated at $1 \times$ $10^{5}$ to $2 \times 10^{5}$ cells $/ \mathrm{mL}$ and treated with MI-3454 or $0.25 \%$ DMSO and cultured at $37^{\circ} \mathrm{C}$ for 7 days. Media were changed on day 4 , with viable cell numbers restored to the original concentration and compounds resupplied. Cell suspensions $(100 \mu \mathrm{L})$ were transferred to 96-well plates for each sample in quadruplicate, followed by an MTT cell proliferation assay (Roche). Plates were read for absorbance at $570 \mathrm{~nm}$ using a PHERAstar BMG microplate reader. The experiments were performed 2 to 3 times in quadruplicate with calculation of mean and SD for each condition.

Generation of HOXA9-MOLM13 cell line. The HOXA9-MOLM13 cell line was generated by transfecting MOLM13 cells with MigR12HA-HOXA9-hpc4-GFP (prepared in-house). After transfection,
GFP-positive cells were sorted out and maintained in 15\% FBS-containing RPMI media. An anti-HoxA9 polyclonal antibody (1:1000, 07-178, Millipore) was used for immunoblotting to validate HoxA9 overexpression. An anti- $\alpha$-tubulin monoclonal antibody (1:5000, T9026, DM1A, Sigma-Aldrich) was used to detect tubulin, which served as a loading control.

Real-time PCR. For real-time quantitative PCR (qRT-PCR) experiments, MV-4-11 and MOLM13 cell lines were plated in 12-well plates $(1 \mathrm{~mL} /$ well $)$ at an initial concentration of $3 \times 10^{5}$ cells $/ \mathrm{mL}$, treated with MI-3454 or $0.25 \%$ DMSO, and incubated at $37^{\circ} \mathrm{C}$ in a $5 \% \mathrm{CO}_{2}$ incubator for 6 days. Media were changed on day 3 of treatment and cell number was adjusted to the initial concentration in each well. The effect of MI-3454 on expression level of target genes in leukemic cells was assessed by qRT-PCR using the approach described previously (19). For qRT-PCR in the primary AML sample 5046, cells were cultured in MethoCult media (H4435, STEMCELL Technologies) at 150,000 cells $/ \mathrm{mL}$ for a total of 17 days with media change on day 10 , followed by cell collection for qRT-PCR analysis. For qRT-PCR studies in bone marrow samples, $1 \times 10^{6}$ to $5 \times 10^{6}$ bone marrow cells/sample were used and the level of gene expression was evaluated in the same way as in leukemic cell lines. For qRT-PCR studies in spleen samples, approximately $3-\mathrm{mm}^{3}$ pieces of spleen were cut and $1 \mathrm{~mL}$ of RNAlater RNA stabilization reagent was added to each sample and samples were stored, before storage at $4^{\circ} \mathrm{C}$. Stabilized tissue was then processed according to the RNeasy Mini Kit protocol (Qiagen). TaqMan primers and probes specific for human genes were used. Information on the TaqMan probes used to quantify expression of individual genes is provided in the Supplemental Methods.

Cytospin/Wright-Giemsa staining. MV-4-11 and MOLM13 cell lines were plated in 12-well plates $(1 \mathrm{~mL} /$ well) at an initial concentration of 3 $\times 10^{5}$ cells $/ \mathrm{mL}$, treated with MI-3454 or $0.25 \%$ DMSO, and incubated at $37^{\circ} \mathrm{C}$ in a $5 \% \mathrm{CO}_{2}$ incubator. Cytospins were prepared as described previously (18).

Colony assays. Details on colony assays are provided in the Supplemental Methods.

Effect of MI-3454 on primary $h C D 34^{+}$cells derived from patient samples. Human $\mathrm{CD}_{34}{ }^{+}$cells sorted out by FACS from primary AML sample 1532 were plated at 100,000 cells/well on MS-5 stromal cells, which were irradiated with $7.5 \mathrm{~Gy}$ and preplated and cultured in MyeloCult medium (H5100 STEMCELL Technologies) supplemented with $20 \mathrm{ng} /$ $\mathrm{mL}$ IL-3, $20 \mathrm{ng} / \mathrm{mL}$ thrombopoietin, $20 \mathrm{ng} / \mathrm{mL} \mathrm{G-CSF}$, and $1 \mu \mathrm{M}$ hydrocortisone. The $\mathrm{CD} 34^{+}$cells from primary sample 1532 were treated with DMSO or MI-3454 at $12 \mathrm{nM}$ and $3 \mathrm{nM}$ for 3 weeks, with media change (supplied with DMSO or MI-3454) once per week. After 3 weeks, cells were washed with PBS and cultured for an additional week without MI-3454 or DMSO. Cells were then collected, counted, and used for flow cytometric analysis of human $\mathrm{CD}^{+} 4^{+}$cells.

PK studies in mice. Details on PK studies are provided in the Supplemental Methods.

Efficacy studies in bioimaging MV-4-11 xenograft model. For studies in the MV-4-11 xenograft model, 8- to 10-week-old female NSG mice (Jackson Laboratory) were intravenously injected with $1 \times 10^{7}$ luciferase-expressing MV-4-11 cells. On day 19 after transplantation, mice were split into vehicle and MI-3454 treatment groups of 5 mice per group and treated for 7 consecutive days with vehicle [20\% (2-hydroxypropyl)- $\beta$-cyclodextrin, $5 \%$ Cremophor] or MI-3454 (120 mg/kg, p.o., b.i.d. and $120 \mathrm{mg} / \mathrm{kg}$, p.o., q.d.). For imaging, mice were kept anes- 
thetized with isoflurane, and $50 \mathrm{mg} / \mathrm{kg}$ luciferase was administered by intraperitoneal injection. Photonic emission was imaged using an IVIS 200 in vivo imaging system (Xenogen) with total imaging time of 10 seconds for each mouse. After 7 days of treatment, bone marrow, spleen, and peripheral blood samples were isolated from mice for analysis. Bone marrow cells were isolated by flushing both femurs and tibias with DMEM containing 5\% FBS. Single-cell cytospins were stained with the HEMA3 Stain Kit (Thermo Fisher Scientific).

Survival studies in MOLM13 xenograft model. Eight- to 10-week-old female NSG mice (Jackson Laboratory) were intravenously injected with $5 \times 10^{5}$ MOLM13 cells. On day 5, mice were split into vehicle and treatment groups of 8 mice per group and were treated for 19 consecutive days with vehicle [20\% (2-hydroxypropyl)- $\beta$-cyclodextrin, $5 \%$ Cremophor] or MI-3454 (100 mg/kg, p.o., b.i.d.). The mice were monitored for leukemia progression and were sacrificed upon signs of distress/disease. Leukemia was confirmed by flow cytometric analysis of hCD $45^{+}$cells using anti-human CD45 antibody (563879, HI30, BD Biosciences), reflecting the level of human leukemic blasts in bone marrow, spleen, and blood samples, as well as by mouse spleen size.

Flow cytometric analysis of mice samples. To assess levels of leukemia burden in the mice transplanted with leukemic cells, cells from bone marrow, peripheral blood, and spleen were suspended in PBS and 1\% FBS for flow cytometric analysis. Red blood cells were lysed with ACK (Lonza). Flow cytometric quantification of hCD $45^{+}$cells from MV-4-11, MOLM13 xenograft models, or PDX models was performed using an anti-human CD45 antibody (563879, HI30, BD Biosciences). All flow cytometry experiments were performed on an LSR II, FACSCanto, or FACSAria (BD Biosciences) and all data were analyzed with FlowJo software (Tree Star, Inc.).

Studies in PDX models of leukemia. Eight- to 10-week-old female NSGS mice (Jackson Laboratory) were intravenously injected with $1 \times$ $10^{7}$ cells from primary patient samples 1532,3055 , and 4392 . For the sample 449 PDX model, cells amplified in the primary recipient mice were used for transplantation $\left(1.3 \times 10^{6}\right)$. After detecting leukemic blasts (hCD $45^{+}$cells) in peripheral blood samples, mice were split into vehicle or MI-3454 treatment groups (5-8 mice/group depending on the PDX model). In the sample 449 PDX model, treatment was initiated 5 days after transplantation owing to the aggressive nature of this model. Mice were treated for 19-21 consecutive days with vehicle or MI-3454 (100 mg/kg, p.o., b.i.d.). Mice were sacrificed upon signs of distress/disease of leukemia or at the endpoint of the experiment. Human blast level was confirmed by flow cytometric analysis of $\mathrm{hCD} 45^{+}$cells in peripheral blood, spleen, and bone marrow, reflecting the progression of leukemia. Survival of mice or level of hCD $45^{+}$cells was used to assess treatment efficacy.

Mouse histology and tissue sample preparation. Details on mouse histology and tissue preparation are provided in the Supplemental Methods.

Analysis of normal hematopoiesis in mice. Bone marrow cells isolated from 8- to 10-week-old female C57BL/6 mice (Taconic Farms) after 19 days of treatment with MI-3454 or vehicle control were prepared as single-cell suspensions in flow cytometry buffer (PBS with $1 \%$ BSA, $10 \mathrm{mM}$ HEPES, $0.01 \% \mathrm{NaN}_{3}$ ), subjected to red blood cell lysis (ACK buffer, Cambrex), and stained on ice with antibodies purchased from BioLegend. To stain LSK cells we used anti-CD150 (115914, TC15-12F12.2), anti-CD48 (103404, HM48-1), anti-Sca-1 (108128, D7), anti-c-Kit (135108, ACK2), and the following lineage cocktail to exclude lineage-positive cells: anti-CD3e (100308, 145-2C11), antiCD8a (100708, 53-6.7), anti-CD11b (101208, M1/70), anti-CD11c (117308, N418), anti-CD19 (115508, 6D5), anti-B220 (103208, RA36B2), anti-TCR $\beta$ (109208, H57-597), anti-TCR $\gamma \delta$ (118108, GL3), antiTer-119 (116206, TER-119), anti-NK1.1 (108708, PK136), and anti-Gr-1 (108408, RB6-8C5). To stain mature cells, we used anti-B220 (103208, RA3-6B2), anti-CD19 (115508, 6D5), anti-IgM (406511, RMM-1), antiCD93 (136510, AA4.1), anti-CD43 (143204, S11), anti-CD11b (101216, M1/70), anti-Gr-1 (108445, RB6-8C5), and anti-Ter-119 (116206, TER-119). Analysis was performed on a FACSAria III (BD Biosciences). Dead cells were excluded with 4',6-diamidino-2-phenylindole (Sigma-Aldrich). Files were analyzed with FlowJo (Tree Star). Statistical analysis was performed with a 2-tailed unpaired Student's $t$ test.

Analysis of stress hematopoiesis in vivo. Details on analysis of MI-3454 on stress hematopoiesis after 5-FU treatment or on hematopoietic reconstitution after transplantation are provided in the Supplemental Methods.

Chemical synthesis of MI-3454. A description of the chemical synthesis of MI-3454 is provided in the Supplemental Methods.

Statistics. Student's $t$ test (unpaired, 2-tailed) or 2-way ANOVA with Tukey's test or 2-way ANOVA with Sidak's multiple-comparisons test or unpaired Welch's $t$ test were used to calculate the significance level between treatment groups, as indicated in figure legends. Significance for survival curves was calculated using log-rank (Mantel-Cox) test. $P$ values of less than 0.05 were considered significant. Graph generation and statistical analysis were performed using GraphPad Prism version 6.02 software.

Accession numbers. Crystallography data for the menin-MI-3454 complex were deposited at the Protein Data Bank under deposition code 605I.

Study approval. All animal experiments in this study were approved by the University Committee on Use and Care of Animals (UCUCA) and the Unit for Laboratory Animal Medicine (ULAM) at the University of Michigan. All mouse studies included randomization and were not blinded.

\section{Author contributions}

SK, HM, and KK designed the experiments, performed the majority of experiments, analyzed data, made figures, and edited the manuscript. SK, TW, HH, and DC designed and synthesized compounds. HM, KK, and EK designed and performed in vivo efficacy studies and biological studies in leukemic cell lines and primary patient samples. TP performed biochemical and cell biology experiments. BML performed crystallography studies. GJ and EP designed and performed in vivo studies of normal hematopoiesis. $\mathrm{MH}, \mathrm{BW}$, and DS designed experiments and generated pharmacokinetics and microsomal stability data. YW and KY designed and performed in vitro profiling studies. SCWL, GDD, WT, MK, AC, OAW, and TG supported the project with primary patient samples and interpreted data. HL and YD prepared cell line used in these studies. MG and LP contributed to the design and discussion of experiments with primary patient samples. SC performed analysis of cytospin slides. IM, LSL, YL, FB, and PR interpreted the data and contributed in writing the manuscript. JG and TC directed the entire project, designed the experiments, analyzed the results, and wrote the manuscript with input from all authors. 


\section{Acknowledgments}

We thank Moshe Talpaz for access to primary patient samples from the Hematological Malignancies Tissue Repositories at the University of Michigan and Martin Carroll from the Stem Cell and Xenograft Core at the University of Pennsylvania for providing additional sets of patient samples. We thank Thomas Look (Dana Farber Cancer Institute) for providing MV-411 cells expressing luciferase. We thank John Bushweller and Olga Guryanova for providing human leukemic cell lines for this project. We thank Andrew Muntean and Qing Li for discussion of a subset of experiments performed within this project. The mouse work was performed under the oversight of the UCUCA at the University of Michigan. This work was funded by Kura Oncology, Inc, NIH R01 grants (1R01CA160467 and 1R01CA201204 to JG and 1R01CA207272 to TC), LLS Scholar award (number 1215-14) to JG, Leukemia \& Lymphoma Society (LLS) Scholar to TC, and an American Cancer Society Mission
Boost grant (MBG-19-095-01-COUN) to JG. GJ is an LLS Fellow. Use of the Advanced Photon Source was supported by the US Department of Energy, Office of Science, Office of Basic Energy Sciences under contract number DE-AC02-06CH11357. Use of the LS-CAT Sector 21 was supported by the Michigan Economic Development Corporation and the Michigan Technology Tri-Corridor for the support of this research program (grant 085P1000817).

Address correspondence to: Jolanta Grembecka, Department of Pathology, University of Michigan, 1150 West Medical Center Drive, MSRB I, Room 4510D, Ann Arbor, Michigan 48108, USA. Phone: 734.615.9319; Email: jolantag@umich.edu. Or to: Tomasz Cierpicki, Department of Pathology, University of Michigan, 1150 West Medical Center Drive, MSRB I, Room 4510C, Ann Arbor, Michigan 48108, USA. Phone: 734.615.9324; Email: tomaszc@umich.edu.
1. Figueroa ME, et al. DNA methylation signatures identify biologically distinct subtypes in acute myeloid leukemia. Cancer Cell. 2010;17(1):13-27.

2. Rodríguez-Paredes M, Esteller M. Cancer epigenetics reaches mainstream oncology. Nat Med. 2011;17(3):330-339.

3. Daigle SR, et al. Selective killing of mixed lineage leukemia cells by a potent small-molecule DOT1L inhibitor. Cancer Cell. 2011;20(1):53-65.

4. Dawson MA, et al. Inhibition of BET recruitment to chromatin as an effective treatment for MLL-fusion leukaemia. Nature. 2011;478(7370):529-533.

5. Zuber J, et al. RNAi screen identifies Brd4 as a therapeutic target in acute myeloid leukaemia. Nature. 2011;478(7370):524-528.

6. Peloquin GL, Chen YB, Fathi AT. The evolving landscape in the therapy of acute myeloid leukemia. Protein Cell. 2013;4(10):735-746.

7. Dimartino JF, Cleary ML. Mll rearrangements in haematological malignancies: lessons from clinical and biological studies. Br J Haematol. 1999;106(3):614-626.

8. Marschalek R. Mechanisms of leukemogenesis by MLL fusion proteins. Br J Haematol. 2011;152(2):141-154.

9. Brown P. Treatment of infant leukemias: challenge and promise. Hematology Am Soc Hematol Educ Program. 2013;2013:596-600.

10. Teachey DT, Hunger SP. Predicting relapse risk in childhood acute lymphoblastic leukaemia. Br J Haematol. 2013;162(5):606-620.

11. Hess JL. MLL: a histone methyltransferase disrupted in leukemia. Trends Mol Med. 2004;10(10):500-507.

12. Krivtsov AV, et al. Transformation from committed progenitor to leukaemia stem cell initiated by MLL-AF9. Nature. 2006;442(7104):818-822.

13. Slany RK. The molecular biology of mixed lineage leukemia. Haematologica. 2009;94(7):984-993.

14. Slany RK. When epigenetics kills: MLL fusion proteins in leukemia. Hematol Oncol. 2005;23(1):1-9.

15. Yokoyama A, Somervaille TC, Smith KS, Rozenblatt-Rosen O, Meyerson M, Cleary ML. The menin tumor suppressor protein is an essential oncogenic cofactor for MLL-associated leukemogenesis. Cell. 2005;123(2):207-218.

16. Yokoyama A, Cleary ML. Menin critically links MLL proteins with LEDGF on cancer-associated target genes. Cancer Cell. 2008;14(1):36-46.

17. Borkin D, et al. Pharmacologic inhibition of the Menin-MLL interaction blocks progression of MLL leukemia in vivo. Cancer Cell. 2015;27(4):589-602.

18. Grembecka J, et al. Menin-MLL inhibitors reverse oncogenic activity of MLL fusion proteins in leukemia. Nat Chem Biol. 2012;8(3):277-284.

19. Shi A, et al. Structural insights into inhibition of the bivalent menin-MLL interaction by small molecules in leukemia. Blood. 2012;120(23):4461-4469.

20. Borkin D, et al. Property focused structure-based optimization of small molecule inhibitors of the protein-protein interaction between menin and mixed lineage leukemia (MLL). J Med Chem. 2016;59(3):892-913.

21. Borkin D, et al. Complexity of blocking bivalent protein-protein interactions: development of a highly potent inhibitor of the menin-mixedlineage leukemia interaction. J Med Chem. 2018;61(11):4832-4850.

22. He S, et al. Menin-MLL inhibitors block oncogenic transformation by MLL-fusion proteins in a fusion partner-independent manner. Leukemia. 2016;30(2):508-513.

23. He S, et al. High-affinity small-molecule inhibitors of the menin-mixed lineage leukemia (MLL) interaction closely mimic a natural protein-protein interaction. JMed Chem. 2014;57(4):1543-1556.

24. Kühn MW, et al. Targeting chromatin regulators inhibits leukemogenic gene expression in NPM1 mutant leukemia. Cancer Discov. 2016;6(10):1166-1181.

25. Falini B, et al. Cytoplasmic nucleophosmin in acute myelogenous leukemia with a normal karyotype. N Engl J Med. 2005;352(3):254-266.

26. Mullighan CG, et al. Pediatric acute myeloid leukemia with NPM1 mutations is characterized by a gene expression profile with dysregulated HOX gene expression distinct from MLL-rearranged leukemias. Leukemia. 2007;21(9):2000-2009.
27. Spencer DH, et al. Epigenomic analysis of the HOX gene loci reveals mechanisms that may control canonical expression patterns in AML and normal hematopoietic cells. Leukemia. 2015;29(6):1279-1289.

28. Alcalay M, et al. Acute myeloid leukemia bearing cytoplasmic nucleophosmin $\left(\mathrm{NPMc}^{+}\right.$ AML) shows a distinct gene expression profile characterized by up-regulation of genes involved in stem-cell maintenance. Blood. 2005;106(3):899-902.

29. Schnittger S, et al. Nucleophosmin gene mutations are predictors of favorable prognosis in acute myelogenous leukemia with a normal karyotype. Blood. 2005;106(12):3733-3739.

30. Papaemmanuil E, et al. Genomic classification and prognosis in acute myeloid leukemia. $N$ Engl JMed. 2016;374(23):2209-2221.

31. Schlenk RF, et al. Mutations and treatment outcome in cytogenetically normal acute myeloid leukemia. NEngl JMed. 2008;358(18):1909-1918.

32. Ostronoff F, et al. Prognostic significance of NPM1 mutations in the absence of FLT3-internal tandem duplication in older patients with acute myeloid leukemia: a SWOG and UK National Cancer Research Institute/Medical Research Council report. JClin Oncol. 2015;33(10):1157-1164.

33. Grembecka J, Belcher AM, Hartley T, Cierpicki T. Molecular basis of the mixed lineage leukemia-menin interaction: implications for targeting mixed lineage leukemias. J Biol Chem. 2010;285(52):40690-40698.

34. Xu S, et al. Design of the first-in-class, highly potent irreversible inhibitor targeting the meninMLL protein-protein interaction. Angew Chem Int Ed Engl. 2018;57(6):1601-1605.

35. Brunetti L, et al. Mutant NPM1 maintains the leukemic state through HOX expression. Cancer Cell. 2018;34(3):499-512.e9.

36. Ernst P, Wang J, Korsmeyer SJ. The role of MLL in hematopoiesis and leukemia. Curr Opin Hematol. 2002;9(4):282-287.

37. McMahon KA, et al. Mll has a critical role in fetal and adult hematopoietic stem cell self-renewal. Cell Stem Cell. 2007;1(3):338-345.

38. Artinger EL, et al. An MLL-dependent network 
sustains hematopoiesis. Proc Natl Acad Sci USA. 2013;110(29):12000-12005.

39. Maillard I, et al. Menin regulates the function of hematopoietic stem cells and lymphoid progenitors. Blood. 2009;113(8):1661-1669.

40. Li BE, Gan T, Meyerson M, Rabbitts TH, Ernst P. Distinct pathways regulated by menin and by MLL1 in hematopoietic stem cells and develop- ing B cells. Blood. 2013;122(12):2039-2046.

41. Caslini C, Yang Z, El-Osta M, Milne TA, Slany RK, Hess JL. Interaction of MLL amino terminal sequences with menin is required for transformation. Cancer Res. 2007;67(15):7275-7283.

42. Li BE, Ernst P. Two decades of leukemia oncoprotein epistasis: the MLL1 paradigm for epigenetic deregulation in leukemia. Exp Hematol.
2014;42(12):995-1012.

43. Daigle SR, et al. Potent inhibition of DOT1L as treatment of MLL-fusion leukemia. Blood. 2013;122(6):1017-1025.

44. Woolthuis CM, et al. Downregulation of MEIS1 impairs long-term expansion of CD34 ${ }^{+}$ NPM1-mutated acute myeloid leukemia cells. Leukemia. 2012;26(4):848-853. 